\title{
Beneficial effects of legumes on parameters of the metabolic syndrome: a systematic review of trials in animal models
}

\author{
Rosario Martínez ${ }^{1}$, María López-Jurado ${ }^{1}$, Carmina Wanden-Berghe ${ }^{2}$, Javier Sanz-Valero ${ }^{3}$, \\ Jesús María Porres ${ }^{1}$ and Garyfallia Kapravelou ${ }^{1 *}$ \\ ${ }^{1}$ Department of Physiology, Institute of Nutrition and Food Technology, University of Granada, Campus Universitario de \\ Cartuja s/n, 18071 Granada, Spain \\ ${ }^{2}$ Universidad CEU Cardenal Herrera, Plaza de Reyes Católicos 19, 03204 Elche, Alicante, Spain \\ ${ }^{3}$ Department of Public Health, History of Science and Gynecology of University Miguel Hernandez of Elche, Avenida de la \\ Universidad, s/n, 03202 Alicante, Spain
}

(Submitted 18 January 2016 - Final revision received 11 April 2016 - Accepted 12 April 2016 - First published online 25 May 2016)

\begin{abstract}
Legume consumption plays a pivotal role in the prevention and treatment of the metabolic syndrome (MetS). This systematic review aimed to highlight the beneficial effects of legume interventions for the prevention and/or improvement of parameters related to the MetS and the implicated metabolic pathways so far reported. The methodology involved a search in four electronic databases (Medline, Web of Science, Scopus, Cochrane Library) from January 2007 to December 2014, considering as descriptors 'Metabolic Syndrome' and 'Fabaceae' and adequately adjusting the equation in each one of them. In total, forty-one studies were finally included. The majority of the studies described a regulating effect on glucose and lipid metabolism due to legume administration, whereas effects on blood pressure and renal parameters are not fully described. Regarding the metabolic pathways involved, they include the up-regulation of genes related to $\beta$-oxidation and acetyl-CoA degradation and the down-regulation of glycolytic and lipogenesis genes, as well as those associated with the acetyl-CoA synthesis. The ameliorating effects of legume consumption on the alterations associated with the MetS are clearly reported and coincide with changes in the expression of protein and genes involved in lipid and glucose metabolism. More research needs to be conducted including more legume species that are highly consumed as part of a healthy dietary pattern.
\end{abstract}

Key words: Metabolic syndrome: Insulin resistance: Fabaceae: Lipolysis: Metabolic pathways

The metabolic syndrome (MetS) represents a clustering of several metabolic disorders among which central obesity and insulin resistance are considered as causative factors ${ }^{(1,2)}$, affecting one-quarter of the world's adult population ${ }^{(3)}$. The initial concept of 'Syndrome X' was described by Reaven ${ }^{(4)}$, whereas the most recent diagnostic criteria, as established by the International Diabetes Federation in $2005^{(5)}$, include obesity (waist circumference $\geq 102 \mathrm{~cm}$ in men or $\geq 88 \mathrm{~cm}$ in women), dyslipidaemia (TAG $\geq 150 \mathrm{mg} / \mathrm{dl}, \mathrm{HDL}<40 \mathrm{mg} / \mathrm{dl}$ in men or $<50 \mathrm{mg} / \mathrm{dl}$ in women), hypertension $(\geq 130 \mathrm{mmHg}$ systolic or $\geq 85 \mathrm{mmHg}$ diastolic) and alterations of glucose metabolism $(>100 \mathrm{mg} / \mathrm{dl} \text {; includes diabetes })^{(6)}$. Although the diagnostic criteria seem to be clear enough, the mechanisms underlying its pathology are not fully understood.

Preventing the development of the MetS requires a multidisciplinary approach, whereas the first step on the treatment of this pathology is focused on the amelioration of the related metabolic alterations and includes mostly lifestyle modifications ${ }^{(7)}$. Nevertheless, in case such modifications prove to be inadequate, the next movement includes the prescription of appropriate pharmacological agents ${ }^{(8)}$. Among lifestyle strategies, low-fat/low-glycaemic-index diets and regular physical exercise are encountered ${ }^{(7)}$. For this reason, legumes have gained increasing interest given that their frequent consumption can help in the control of lipid homoeostasis and, consequently, reduce the risk of CVD. In addition, their consumption is associated with a better glycaemic control in diabetic patients and has exhibited hypolipidaemic effects by reducing the absorption of cholesterol. Their contribution to weight management because of their beneficial effect on appetite-regulating hormones and satiety has also been demonstrated ${ }^{(9,10)}$.

The bioactive compounds that legumes contain such as resistant starch, $\alpha$ galactoside oligosaccharides, phytate, polyphenols and saponins may act as potential physiological modulators of metabolism, given that they inhibit the activity

Abbreviation: MetS, metabolic syndrome.

* Corresponding author: G. Kapravelou, fax +34958248 959, email gkapravelou@gmail.com 
of angiotensin-converting enzyme and exhibit prebiotic effects, as well as antioxidant and bile acid-binding properties ${ }^{(11,12)}$, thus showing promising potential as functional ingredients.

Taking into account that the actual lifestyle is at the same time leading to the increase of the prevalence of risk factors that induce the MetS and the undervalued consumption of legume foodstuff, as reflected by epidemiological nutritional surveys, there is a clear need to reinforce lifestyle strategies in order to better prevent the development of the MetS. The present review aimed at gathering the outcomes of recent intervention studies by putting together the beneficial effects that the consumption of different legumes exert on different alterations associated with the MetS.

\section{Methods}

\section{Study eligibility}

Considering that the aim of the present review was to collect the most recent and representative data for the effects of the legumes on the MetS, we performed a bibliometric analysis in the field of nutrition, which established the period of 7 years as the obsolescence period of the results of these studies ${ }^{(13)}$. This period assured that more than half of the actual scientific production would be included (Burton-Kebler index: obsolescence according to median age/median production) ${ }^{(14)}$. Therefore, the cut-off point for the publication date was established from January 2007 to December 2014. Although the present review focused on collecting data of animal trials, no filters were used at this point in order to prevent losing any entry not properly registered. Therefore, further exclusion of the entries was performed manually.

Thus far, the eligibility of the publications was confirmed by fulfilling the following inclusion criteria:

- The research articles should be recent intervention studies published after the year 2007, in which consumption of legume or administration of the legume-derived product was tested against different alterations related to the MetS.

- The research articles should be published in peer review journals, and the ones with complete text access were selected.

\section{Data sources}

A comprehensive and systematic review of literature was conducted using four electronic databases: MedLars Online International Literature, via PubMed ${ }^{\circledR}$, Web of Science, SCOPUS and the Cochrane Library Plus. The first step included the definition of the search terms through the use of Medical Subject Headings (MeSH) and considering as descriptors 'Metabolic Syndrome' and 'Fabaceae', in all the possible forms used by the indexed publications in PubMed. The final equation was ('Metabolic Syndrome X'[Mesh] OR 'Metabolic Syndrome X'[Title/Abstract] OR 'Metabolic Syndrome'[Title/ Abstract] OR 'Insulin Resistance Syndrome X'[Title/Abstract] OR 'Syndrome X, Metabolic'[Title/Abstract] OR 'Syndrome X, Insulin Resistance'[Title/Abstract] OR 'Metabolic X Syndrome'[Title/Abstract]
OR 'Syndrome, Metabolic X'Title/Abstract] OR 'X Syndrome, Metabolic'[Title/Abstract] OR 'Dysmetabolic Syndrome X'TTitle/ Abstract] OR 'Syndrome X, Dysmetabolic'[Title/Abstract] OR 'Reaven Syndrome X'Title/Abstract] OR 'Syndrome X, Reaven'[Title/Abstract] OR 'Metabolic Cardiovascular Syndrome'[Title/ Abstract] OR 'Cardiovascular Syndrome, Metabolic'[Title/Abstract] OR 'Cardiovascular Syndromes, Metabolic'[Title/Abstract] OR 'Syndrome, Metabolic Cardiovascular'[Title/Abstract]) AND ('Fabaceae'[Mesh] OR 'Leguminosae'[Title/Abstract] OR 'Legume'[Title/Abstract] OR 'Legumes'[Title/Abstract] OR 'Beans'[Title/ Abstract] OR 'Amorpha'[Title/Abstract] OR 'Andira'[Title/Abstract] OR 'Baptisia'[Title/Abstract] OR 'Callerya'[Title/Abstract] OR 'Ceratonia'[Title/Abstract] OR 'Clathrotropis'[Title/Abstract] OR 'Colophospermum'[Title/Abstract] OR 'Copaifera'[Title/Abstract] OR 'Delonix'[Title/Abstract] OR 'Euchresta'[Title/Abstract] OR 'Guibourtia'[Title/Abstract] OR 'Machaerium'[Title/Abstract] OR 'Pithecellobium'[Title/Abstract] OR 'Pithecolobium'[Title/Abstract] OR 'Stryphnodendron'[Title/Abstract] OR 'Tachigalia'[Title/ Abstract] OR 'Afzelia'[Title/Abstract]). The same search strategy was applied for the other three databases, and the equation was suitably adapted. The repeated studies found in the different databases were considered only once in the total list of the studies. The list of eligible studies was completed by the search in the reference list of the publications selected and respecting the a priori inclusion criteria established.

\section{Study selection}

Two of the authors (R. M. and G. K.) carried out the first screening of the eligible studies separately, which included the review of the abstracts of the studies and the selection of the suitable ones for full-text examination. At this point, bibliographic reviews, epidemiological studies, editorials, case reports and book chapters were excluded. There were no language restrictions. At the second stage of the selection process, the same authors examined the full-text articles and then selected the adequate studies to include. As the aim of the study was to review the existing data on animal intervention studies, the two authors manually excluded the clinical trials in humans. The decisions for the inclusion/exclusion were taken following mutual discussion and consensus. If consensus was not possible, two (M. L.-J. and J. M. P.) more authors examined the articles and the consensus was established after the discussion between the four authors.

\section{Data extraction}

After the conclusion of the study selection process, R. M. and G. K. independently reviewed and extracted the data of the selected studies. The overall inter-rater agreement rate before correcting discrepant items was determined using Cohen's $\kappa$ statistic $^{(15)}$ and established to be superior to $0 \cdot 80^{(16)}$. Any discrepancies were resolved after consensus between the two or four authors (R. M. and G. K.) or between the four of them (including M. L.-J. and J. M. P.) if necessary. The quality of the studies selected was determined by the use of a specific questionnaire for the clinical trials (Scientific studies-clinical trials quality-evaluation questionnaire, CACEC-EC), which is 


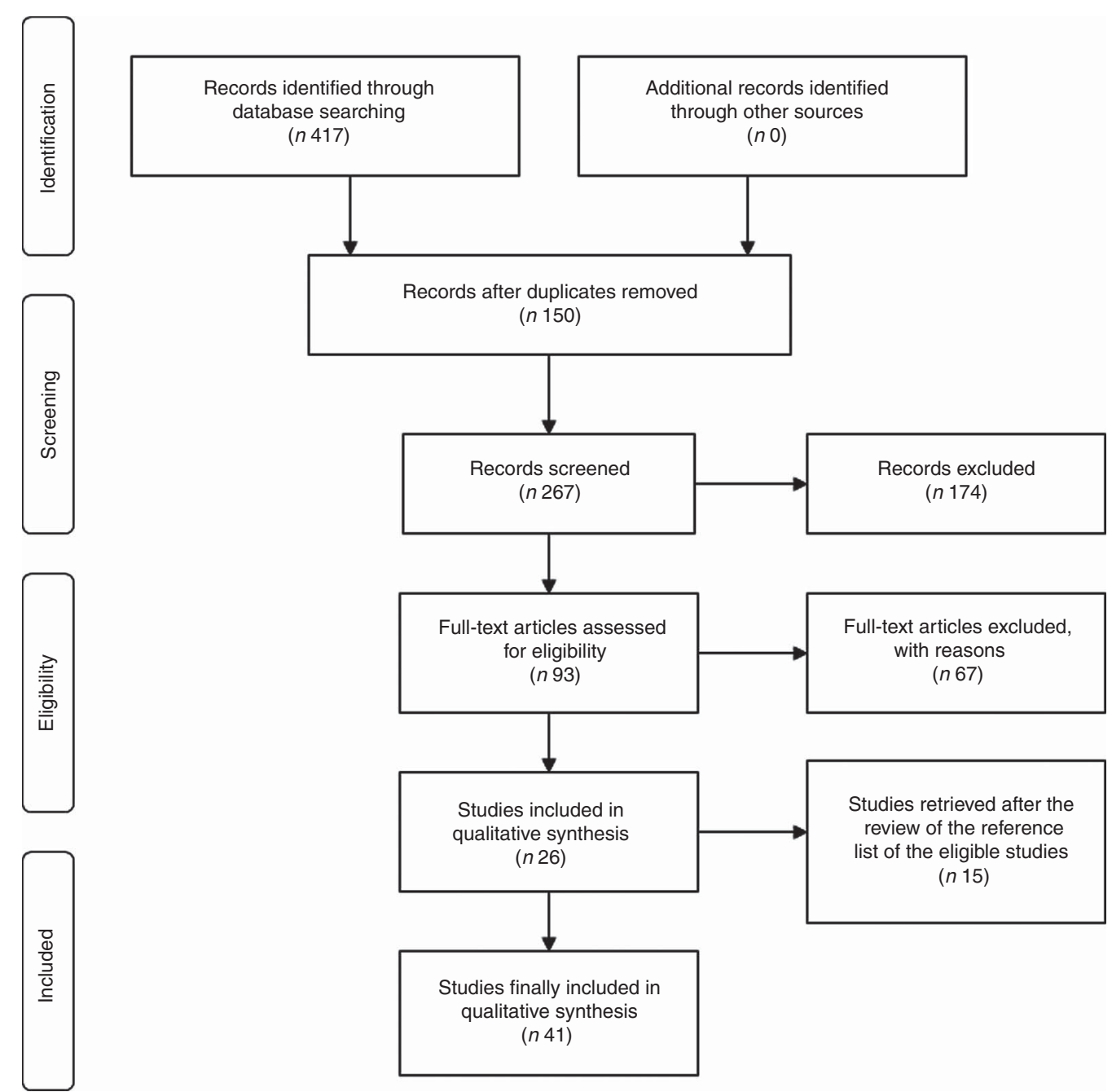

Fig. 1. Flow diagram of the eligible studies included in the systematic review.

divided into two parts: the first part includes filter questions that determine whether the study fulfils the methodology premises of a clinical trial (score $>6$ ) and the second part finally determines the quality (0-6, low; 7-14, good; $15-20$, excellent) of the study in its different parts (intervention, sample manipulation, results and conclusions).

The extracted data were grouped in a table and classified according to the legume studied. In the different columns, the reference of the publication, the animal model (number, age and type of animals, experimental groups) used, the intervention (legume type and quantity consumed, technological process and experimental period) followed and the principal beneficial results achieved are noted, in order to facilitate the comprehension of the selected studies.

\section{Results}

The initial systematic search in the different electronic databases resulted in 417 references. After the exclusion of duplicated references ( $n$ 150, among which forty-three clinical trials, forty-nine epidemiological studies, fifty-eight reviews), there were 267 potentially eligible studies remaining. The first screening resulted in exclusion of bibliographic reviews ( $n$ 92), epidemiological studies ( $n$ 76) and other types of studies such as book chapters, case reports or editorials ( $n 6$ in total). The possibly eligible studies were then reduced to ninety-three. The second screening, which was manually performed, resulted in the exclusion of: trials that studied parameters not relevant with the MetS alterations ( $n$ 27); clinical trials performed in humans ( $n 30)$; in vitro studies ( $n$ 8); or finally, animal studies that used legume diet intervention but obtained only negative results due to the specific intervention. After the second screening, twenty-six eligible studies remained, to which fifteen new were added after reviewing the reference lists of the studies already selected. After the whole process was completed, we retracted forty-one eligible studies, which included only in vivo experiments in different experimental animal models making use of a legume as part of the diet intervention. The entire process followed is represented in Fig. 1. In total, sixteen different legumes were reported in these studies. The beneficial effects on several parameters of the MetS were collected and are presented in Tables 1 and 2 . 
Table 1. Beneficial effects of legumes on several parameters of the metabolic syndrome

\begin{tabular}{|c|c|c|c|c|c|c|c|c|c|}
\hline \multirow[b]{2}{*}{ References } & \multirow[b]{2}{*}{ Animal models } & \multirow[b]{2}{*}{ Intervention } & \multicolumn{7}{|c|}{ Beneficial effects on } \\
\hline & & & $\begin{array}{l}\text { Glucose } \\
\text { metabolism }\end{array}$ & $\begin{array}{l}\text { Lipid } \\
\text { metabolism }\end{array}$ & $\begin{array}{l}\text { Blood } \\
\text { pressure }\end{array}$ & $\begin{array}{c}\text { BW/ } \\
\text { composition }\end{array}$ & $\begin{array}{l}\text { Inflammation } \\
\text { markers }\end{array}$ & $\begin{array}{l}\text { Oxidative } \\
\text { damage }\end{array}$ & $\begin{array}{l}\text { Renal } \\
\text { function }\end{array}$ \\
\hline \multicolumn{10}{|l|}{ Glycine max/soyabean } \\
\hline Potu et al. ${ }^{(21)}$ & $\begin{array}{l}\text { M: female Ossabaw pigs } \\
\text { A/W: } 3 \text { months }\end{array}$ & $\begin{array}{l}\text { LA: SBO and LLO } \\
\text { EP: } 8 \text { weeks }\end{array}$ & - & - & - & - & $\sqrt{ }$ & - & - \\
\hline Mori et al. ${ }^{(19)}$ & $\begin{array}{l}\text { M: male Wistar rats } \\
\text { A/W: } 6 \text { weeks }\end{array}$ & $\begin{array}{l}\text { LA: PL from soyabeans } \\
\text { EP: } 10 \text { weeks }\end{array}$ & $\sqrt{ }$ & $\sqrt{ }$ & - & - & - & - & - \\
\hline Palanisamy et al. ${ }^{(26)}$ & $\begin{array}{l}\text { M: male Wistar rats MS } \\
\text { A/W: } 150-160 \mathrm{~g}\end{array}$ & $\begin{array}{l}\text { LA: FSD } \\
\text { EP: } 60 \mathrm{~d}\end{array}$ & $\sqrt{ }$ & - & $\sqrt{ }$ & $\sqrt{ }$ & - & $\sqrt{ }$ & $\sqrt{ }$ \\
\hline Ronis et al. ${ }^{(17)}$ & $\begin{array}{l}\text { M: Sprague-Dawley rats } \\
\text { A/W: - }\end{array}$ & $\begin{array}{l}\text { LA: SPI; SPI+; SPI- } \\
\text { Expt 1: } 33 \mathrm{~d}\end{array}$ & $\sqrt{ }$ & $\sqrt{ }$ & - & $\sqrt{ }$ & - & - & - \\
\hline Nordentoft et al. ${ }^{(20)}$ & $\begin{array}{l}\text { M: male KK-A Y and non-diabetic } \\
\text { C57/BL mice } \\
\text { A/W: } 5 \text { weeks }\end{array}$ & $\begin{array}{l}\text { LA: SBP } \\
\text { EP: } 9 \text { weeks }\end{array}$ & $\sqrt{ }$ & $\sqrt{ }$ & - & $\sqrt{ }$ & - & - & - \\
\hline Wagner et al. ${ }^{(23)}$ & $\begin{array}{l}\text { M: male monkey and obese, } \\
\text { hyperinsulinaemic monkey } \\
\text { A/W: adult and } 8 \text { years }\end{array}$ & $\begin{array}{l}\text { LA: SPI and whole SOY } \\
\text { EP: Expt 1: } 25 \text { months; Expt 2: } \\
\quad 40 \text { weeks }\end{array}$ & - & $\sqrt{ }$ & - & - & - & - & - \\
\hline Hwang et al. ${ }^{(27)}$ & $\begin{array}{l}\text { M: obese and lean male Zucker rats } \\
\text { A/W: } 5 \text { weeks }\end{array}$ & $\begin{array}{l}\text { LA: SP } \\
\text { EP: } 8 \text { weeks }\end{array}$ & - & - & - & - & - & - & $\sqrt{ }$ \\
\hline $\begin{array}{l}\text { Torre-Villalvazo } \\
\text { et al. }\end{array}$ & $\begin{array}{l}\text { M: male Sprague-Dawley rats } \\
\text { A/W: } 4 \text { weeks }\end{array}$ & $\begin{array}{l}\text { LA: SP } \\
\text { EP: } 180 \mathrm{~d}\end{array}$ & - & $\sqrt{ }$ & - & - & - & - & - \\
\hline Davis et al. ${ }^{(24)}$ & $\begin{array}{l}\text { M: obese male Zucker diabetic fatty } \\
\left(\mathrm{ZDF} / \mathrm{Lepr}^{\mathrm{fa}}\right) \text { rats } \\
\text { A/W: } 6 \text { weeks }\end{array}$ & $\begin{array}{l}\text { LA: SP } \\
\text { EP: } 11 \text { weeks }\end{array}$ & $\sqrt{ }$ & $\sqrt{ }$ & - & $\sqrt{ }$ & - & - & $\sqrt{ }$ \\
\hline Zhou et al. ${ }^{(25)}$ & $\begin{array}{l}\text { M: FVB/N mice } \\
\text { A/W: } 5-6 \text { weeks }\end{array}$ & $\begin{array}{l}\text { LA: SPIs; SPC; } \\
\text { EP: } 8 \text { weeks }\end{array}$ & $\sqrt{ }$ & - & - & $\sqrt{ }$ & - & - & - \\
\hline $\begin{array}{l}\text { Barrios-Ramos } \\
\quad \text { et al. }{ }^{(18)}\end{array}$ & $\begin{array}{l}\text { M: male Wistar rats } \\
\text { A/W: } 250-260 \mathrm{~g}\end{array}$ & $\begin{array}{l}\text { LA: oat, soyabean, cocoa, } \\
\text { fish oil } \\
\text { EP: } 14 \text { weeks }\end{array}$ & $\sqrt{ }$ & $\sqrt{ }$ & $\sqrt{ }$ & - & - & - & - \\
\hline \multicolumn{10}{|c|}{ Trigonella foenum graecum/enugreek } \\
\hline Muraki et al. ${ }^{(28)}$ & $\begin{array}{l}\text { M: male Sprague-Dawley rats } \\
\text { A/W: } 3 \text { weeks }\end{array}$ & $\begin{array}{l}\text { LA: FSP } \\
\text { EP: } 12 \text { weeks }\end{array}$ & $\sqrt{ }$ & - & - & - & - & - & - \\
\hline $\begin{array}{l}\text { Belguith-Hadriche } \\
\text { et al. }{ }^{(30)}\end{array}$ & $\begin{array}{l}\text { M: male Wistar rat } \\
\text { A/W: } 120 \mathrm{~g}\end{array}$ & $\begin{array}{l}\text { LA: EAES } \\
\text { EP }\end{array}$ & - & $\sqrt{ }$ & - & - & - & $\sqrt{ }$ & - \\
\hline $\begin{array}{l}\text { Kannappan \& } \\
\quad \text { Anuradha }^{(33)}\end{array}$ & $\begin{array}{l}\text { M: male Wistar rats } \\
\text { A/W: } 150-180 \mathrm{~g}\end{array}$ & $\begin{array}{l}\text { LA: FPEt } \\
\text { EP: } 45 \mathrm{~d}\end{array}$ & $\sqrt{ }$ & - & - & - & - & - & - \\
\hline Ramadan et al. ${ }^{(29)}$ & $\begin{array}{l}\text { M: male Wistar rats. Diabetes, obese } \\
\text { and immunosuppressive } \\
\text { A/W: } 125-135 \mathrm{~g}\end{array}$ & $\begin{array}{l}\text { LA: FSP } \\
\text { EP: } 4 \text { weeks }\end{array}$ & $\sqrt{ }$ & $\sqrt{ }$ & - & $\sqrt{ }$ & $\sqrt{ }$ & $\sqrt{ }$ & - \\
\hline Mowla et al. ${ }^{(32)}$ & $\begin{array}{l}\text { M: male Wistar rats } \\
\text { A/W: } 150-250 \mathrm{~g}\end{array}$ & $\begin{array}{l}\text { LA: ethanolic seed extract } \\
\text { EP: } 2 \mathrm{~h}\end{array}$ & $\sqrt{ }$ & - & - & - & - & - & - \\
\hline $\begin{array}{l}\text { Srichamroen } \\
\text { et al. }\end{array}$ & $\begin{array}{l}\text { M: male Sprague-Dawley rats } \\
\text { A/W: } 175-200 \mathrm{~g}\end{array}$ & $\begin{array}{l}\text { LA: GAL from seeds } \\
\text { EP: } 3 \text { weeks }\end{array}$ & $\sqrt{ }$ & $\sqrt{ }$ & - & $\sqrt{ }$ & - & - & - \\
\hline $\begin{array}{l}\text { Srichamroen } \\
\quad \text { et al. }{ }^{(35)}\end{array}$ & $\begin{array}{l}\text { M: genetically lean and obese } \\
\text { JCR rats } \\
\text { A/W: } 4 \text { months }\end{array}$ & $\begin{array}{l}\text { LA: GAL } \\
\text { EP: } 14 \mathrm{~d}\end{array}$ & $\sqrt{ }$ & - & - & - & - & - & - \\
\hline Eidi et al. ${ }^{(31)}$ & $\begin{array}{l}\text { M: male Wistar rats } \\
\text { A/W: } 200-250 \mathrm{~g}\end{array}$ & $\begin{array}{l}\text { LA: ethanolic extract from } \\
\text { seeds } \\
\text { EP: } 14 \mathrm{~d}\end{array}$ & $\sqrt{ }$ & $\sqrt{ }$ & - & - & $\sqrt{ }$ & - & $\sqrt{ }$ \\
\hline
\end{tabular}




\section{N British Journal of Nutrition}

\begin{tabular}{|c|c|c|c|c|c|c|c|c|c|}
\hline \multirow[b]{2}{*}{ References } & \multirow[b]{2}{*}{ Animal models } & \multirow[b]{2}{*}{ Intervention } & \multicolumn{7}{|c|}{ Beneficial effects on } \\
\hline & & & $\begin{array}{l}\text { Glucose } \\
\text { metabolism }\end{array}$ & $\begin{array}{l}\text { Lipid } \\
\text { metabolism }\end{array}$ & $\begin{array}{l}\text { Blood } \\
\text { pressure }\end{array}$ & $\begin{array}{c}\text { BW/ } \\
\text { composition }\end{array}$ & $\begin{array}{l}\text { Inflammation } \\
\text { markers }\end{array}$ & $\begin{array}{l}\text { Oxidative } \\
\text { damage }\end{array}$ & $\begin{array}{l}\text { Renal } \\
\text { function }\end{array}$ \\
\hline \multicolumn{10}{|c|}{ Phaseolus vulgaris/beans } \\
\hline Zaru et al. ${ }^{(39)}$ & $\begin{array}{l}\text { M: male Wistar rats } \\
\text { A/W: } 300 \mathrm{~g}\end{array}$ & $\begin{array}{l}\text { LA: } P v \text { and } C s \\
\text { EP: } 17 \mathrm{~d}\end{array}$ & $\sqrt{ }$ & - & - & - & - & - & - \\
\hline $\begin{array}{l}\text { Adel \& } \\
\text { El-shinnawy }{ }^{(36)}\end{array}$ & $\begin{array}{l}\text { M: male Wistar rats } \\
\text { A/W: } 150-160 \mathrm{~g}\end{array}$ & $\begin{array}{l}\text { LA: beans. Hulls, fibre MCC } \\
\text { EP: } 10 \mathrm{~d}\end{array}$ & $\sqrt{ }$ & $\sqrt{ }$ & - & $\sqrt{ }$ & - & - & - \\
\hline Zhu et al. ${ }^{(38)}$ & $\begin{array}{l}\text { M: female Sprague-Dawley rats and } \\
\text { C57BL/6J obese male mice } \\
\text { A/W: } 19 \text { and } 27 \mathrm{~d}\end{array}$ & $\begin{array}{l}\text { LA: dry red bean } \\
\text { EP: } 15,7,12 \mathrm{~d} \text { and } 7 \text { weeks }\end{array}$ & - & $\sqrt{ }$ & - & $\sqrt{ }$ & - & - & - \\
\hline Carai et al. ${ }^{(37)}$ & $\begin{array}{l}\text { M: male Zucker fa/fa rats } \\
\text { A/W: } 525 \mathrm{~g}\end{array}$ & $\begin{array}{l}\text { LA: dry extract } \\
\text { EP: } 5 \mathrm{~d}\end{array}$ & $\sqrt{ }$ & - & - & $\sqrt{ }$ & - & - & - \\
\hline \multicolumn{10}{|c|}{ Vigna angularis/adzuki beans } \\
\hline $\begin{array}{l}\text { Kitano-Okada } \\
\quad \text { et }^{\text {al. }}{ }^{(42)}\end{array}$ & $\begin{array}{l}\text { M: male Fischer } 44 \text { rats } \\
\text { A/W: } 7 \text { weeks }\end{array}$ & $\begin{array}{l}\text { LA: } 1 \% \mathrm{w} / \mathrm{w} \text { bean extract } \\
\text { EP: } 4 \text { weeks }\end{array}$ & - & $\sqrt{ }$ & - & - & - & - & - \\
\hline Itoh \& Furuichi ${ }^{(40)}$ & $\begin{array}{l}\text { M: KK-A Y mice } \\
\text { A/W: } 5,8 \text { and } 3 \text { weeks }\end{array}$ & $\begin{array}{l}\text { LA: CEL or EtEx: } \mathrm{mg} / \mathrm{kg} \text { per } \mathrm{d} \\
\text { EP: } 7,4 \text { and } 1 \text { weeks }\end{array}$ & - & $\sqrt{ }$ & - & - & - & - & - \\
\hline Itoh et al. ${ }^{(41)}$ & $\begin{array}{l}\text { M: male Sprague-Dawley rats } \\
\text { A/W: } 5 \text { weeks } / 40-60 \mathrm{~g}\end{array}$ & $\begin{array}{l}\text { LA: adzuki bean extract } \\
\text { EP: } 2 \text { weeks }\end{array}$ & $\sqrt{ }$ & $\sqrt{ }$ & - & - & - & - & $\sqrt{ }$ \\
\hline \multicolumn{10}{|c|}{ Pissum sativum/yellow pea } \\
\hline Eslinger et al. ${ }^{(43)}$ & $\begin{array}{l}\text { M: male Sprague-Dawley induced } \\
\text { obesity. } \\
\text { A/W: } 5 \text { weeks }\end{array}$ & $\begin{array}{l}\text { LA: yellow pea-derived } \\
\text { fractions } \\
\text { OFS, PF, PFL and PS } \\
\text { EP: } 6 \text { weeks }\end{array}$ & $\sqrt{ }$ & $\sqrt{ }$ & - & $\sqrt{ }$ & - & - & - \\
\hline Marinangeli et al. ${ }^{(44)}$ & $\begin{array}{l}\text { M: male golden Syrian hamsters } \\
\text { A/W: } 2 \text { weeks }\end{array}$ & $\begin{array}{l}\text { LA: yellow pea } \\
\text { EP: } 28 \mathrm{~d}\end{array}$ & $\sqrt{ }$ & - & - & - & - & - & - \\
\hline \multicolumn{10}{|c|}{ Astragalus membranaceus } \\
\hline Gao et al..$^{(45)}$ & $\begin{array}{l}\text { M: male prediabetic rats } \\
\text { A/W: } 8 \text { weeks } / 170-190 \mathrm{~g}\end{array}$ & $\begin{array}{l}\text { LA: saponins from roots; JQ-R } \\
\text { EP: } 4 \text { weeks }\end{array}$ & $\sqrt{ }$ & $\sqrt{ }$ & - & $\sqrt{ }$ & - & - & - \\
\hline Hoo et al..$^{(46)}$ & $\begin{array}{l}\text { M: male C57BL/KsJ db/db } \\
\text { A/W: } 10 \text { weeks }\end{array}$ & $\begin{array}{l}\text { LA: dry root }(\mathrm{Rx}, 2 \mathrm{~g} / \mathrm{kg} \text { per d) } \\
\text { EP: } 12 \text { weeks }\end{array}$ & $\sqrt{ }$ & $\sqrt{ }$ & - & - & $\sqrt{ }$ & - & - \\
\hline \multicolumn{10}{|l|}{ Glycyrrhiza glabra } \\
\hline Yoke et al. ${ }^{(48)}$ & $\begin{array}{l}\text { M: male Sprague-Dawley rats } \\
\text { A/W: } 6 \text { weeks }\end{array}$ & $\begin{array}{l}\text { LA: GA } 100 \mathrm{mg} / \mathrm{kg} \\
\text { EP: } 24 \mathrm{~h}\end{array}$ & $\sqrt{ }$ & $\sqrt{ }$ & - & - & - & - & - \\
\hline Aoki et al. (47) $^{-}$ & $\begin{array}{l}\text { M: female } \mathrm{C} 7 \mathrm{BL} / 6 \mathrm{~J} \text { mice } \\
\text { A/W: } 18 \text { weeks }\end{array}$ & $\begin{array}{l}\text { LA: LFO } \\
\text { EP: } 8 \text { weeks HFD }+8 \text { weeks } \\
\text { LFO }\end{array}$ & $\sqrt{ }$ & $\sqrt{ }$ & - & $\sqrt{ }$ & - & - & - \\
\hline \multicolumn{10}{|l|}{ Other legumes } \\
\hline $\begin{array}{l}\text { Beltrán-Debón } \\
\quad \text { et al. }{ }^{(60)}\end{array}$ & $\begin{array}{l}\text { M: C57BL/6J male mice } \\
\text { A/W: } 10 \text { weeks }\end{array}$ & $\begin{array}{l}\text { LA: Aspalathus linearis extracts } \\
\text { EP: } 14 \text { weeks }\end{array}$ & - & $\sqrt{ }$ & - & - & - & - & - \\
\hline Dai et al. ${ }^{(52)}$ & $\begin{array}{l}\text { M: male Syrian Hamsters } \\
\text { A/W: } 4 \text { weeks }\end{array}$ & $\begin{array}{l}\text { LA: Cajanus cajan } \mathrm{L} . \\
\quad(\text { Pigeon pea) } \\
\text { EP: } 8 \text { weeks }\end{array}$ & - & $\sqrt{ }$ & - & - & - & $\sqrt{ }$ & - \\
\hline Tzeng et al. ${ }^{(58)}$ & $\begin{array}{l}\text { M: 3T3-L1 adipocytes/old Wistar rats } \\
\text { A/W: } 8 \text { weeks }\end{array}$ & $\begin{array}{l}\text { LA: Cassia tora } \\
\text { Seeds: ethanol extract } \\
\text { EP: } 8 \text { weeks }\end{array}$ & - & $\sqrt{ }$ & - & $\sqrt{ }$ & - & - & - \\
\hline
\end{tabular}




\section{N British Journal of Nutrition}

Table 1. Continued

\begin{tabular}{|c|c|c|c|c|c|c|c|c|c|}
\hline \multirow[b]{2}{*}{ References } & \multirow[b]{2}{*}{ Animal models } & \multirow[b]{2}{*}{ Intervention } & \multicolumn{7}{|c|}{ Beneficial effects on } \\
\hline & & & $\begin{array}{l}\text { Glucose } \\
\text { metabolism }\end{array}$ & $\begin{array}{l}\text { Lipid } \\
\text { metabolism }\end{array}$ & $\begin{array}{l}\text { Blood } \\
\text { pressure }\end{array}$ & $\begin{array}{c}\text { BW/ } \\
\text { composition }\end{array}$ & $\begin{array}{l}\text { Inflammation } \\
\text { markers }\end{array}$ & $\begin{array}{l}\text { Oxidative } \\
\text { damage }\end{array}$ & $\begin{array}{l}\text { Renal } \\
\text { function }\end{array}$ \\
\hline Weidner et al. ${ }^{(56)}$ & $\begin{array}{l}\text { M: male C57BL/6 mice; leptin } \\
\text { receptor-deficient } \mathrm{db} / \mathrm{db} \text { mice and } \\
\text { male C57BL/6 } \\
\text { A/W: } 6,9 \text { and } 9 \text { weeks }\end{array}$ & $\begin{array}{l}\text { LA: Glycyrrhiza foetida } \\
\text { Amorpha fruticosa } \\
\text { EP: } 3 \text { weeks; } 3 \text { and } 15 \text { weeks }\end{array}$ & $\sqrt{ }$ & $\sqrt{ }$ & - & $\sqrt{ }$ & $\sqrt{ }$ & - & - \\
\hline Boualga et al. ${ }^{(61)}$ & $\begin{array}{l}\text { M: male Wistar rats } \\
\text { A } / \mathrm{W}: 60-70 \mathrm{~g}\end{array}$ & $\begin{array}{l}\text { LA: Lens culinaris/Cicer } \\
\quad \text { arietinum } \\
\text { LP/CPr } \\
\text { EP: } 28 \mathrm{~d}\end{array}$ & - & $\sqrt{ }$ & - & $\sqrt{ }$ & - & - & - \\
\hline Okwuosa et al. ${ }^{(53)}$ & $\begin{array}{l}\text { M: male albino Wistar rats } \\
\text { A/W: } 100-130 \mathrm{~g}\end{array}$ & $\begin{array}{l}\text { LA: Pterocarpus santaniloides } \\
\text { AEPS } \\
\text { MEPS } \\
\text { EP: } 10 \mathrm{~d}\end{array}$ & $\sqrt{ }$ & $\sqrt{ }$ & - & - & - & - & - \\
\hline Peng et al. ${ }^{(54)}$ & $\begin{array}{l}\text { M: female pups of SP-SHR } \\
\text { A/W: } 4 \text { weeks }\end{array}$ & $\begin{array}{l}\text { LA: Pueraria lobata (kudzu) } \\
\text { EP: } 2 \text { months }\end{array}$ & $\sqrt{ }$ & $\sqrt{ }$ & $\sqrt{ }$ & - & - & - & - \\
\hline Shahraki et al. ${ }^{(55)}$ & $\begin{array}{l}\text { M: male Wistar rats } \\
\text { A/W: } 130-150 \mathrm{~g}\end{array}$ & $\begin{array}{l}\text { LA: Tamarindus indica } \\
\text { Seed: aqueous extract } \\
\text { EP: } 8 \text { weeks }\end{array}$ & - & $\sqrt{ }$ & - & $\sqrt{ }$ & - & - & - \\
\hline Pavana et al. ${ }^{(57)}$ & $\begin{array}{l}\text { M: albino Wistar male rats: induced } \\
\text { DM by streptozotocin } \\
\text { A/W: } 150-200 \mathrm{~g}\end{array}$ & $\begin{array}{l}\text { LA: Tephrosia purpurea leaves } \\
\text { (TpALet) } \\
\text { EP: } 45 \mathrm{~d}\end{array}$ & $\sqrt{ }$ & $\sqrt{ }$ & - & - & - & - & - \\
\hline
\end{tabular}

BW, body weight; M, model; A/W, age/weight; LA, legume administration; SBO, soyabean oil; LLO, low a-linolenic soyabean oil; EP, experimental period; -, no effect; $\sqrt{ }$, positive effect; FSD, soya protein concentrate; SPI, soya protein isolate; SBP, high content isoflavone soya protein; SP, soya protein; SPIs, isoflavone-depleted soya protein isolates; SPC, soya phytochemicals extract; FSP, fenugreek seed powder; EAES, ethyl acetate extract from seeds; FPEt, polyphenols from seeds; GAL, galactomannan; MCC, mycrocrystalline cellulose; CEL, cellulose; OFS, oligofructose; PF, yellow pea fibre; PFL, yellow pea flour; PS, yellow pea starch; JQ-R, refined JinQi-JiangTang tablet; GA, glycyrrhizic acid; LFO, licorice flavonoid oil; HFD, high-fat diet; LP, lentil protein; CPr, chickpea protein; AEPS, aqueous extract of Pterocarpus santaniloides; MEPS, methanolic extract of Pterocarpus santaniloide; SP-SHR, stroke prone - spontaneously hypertensive rat. 
Table 2. Beneficial effects of legumes on different parameters of the metabolic syndrome expressed as numerical data (Mean values and standard deviations; mean values with their standard errors)

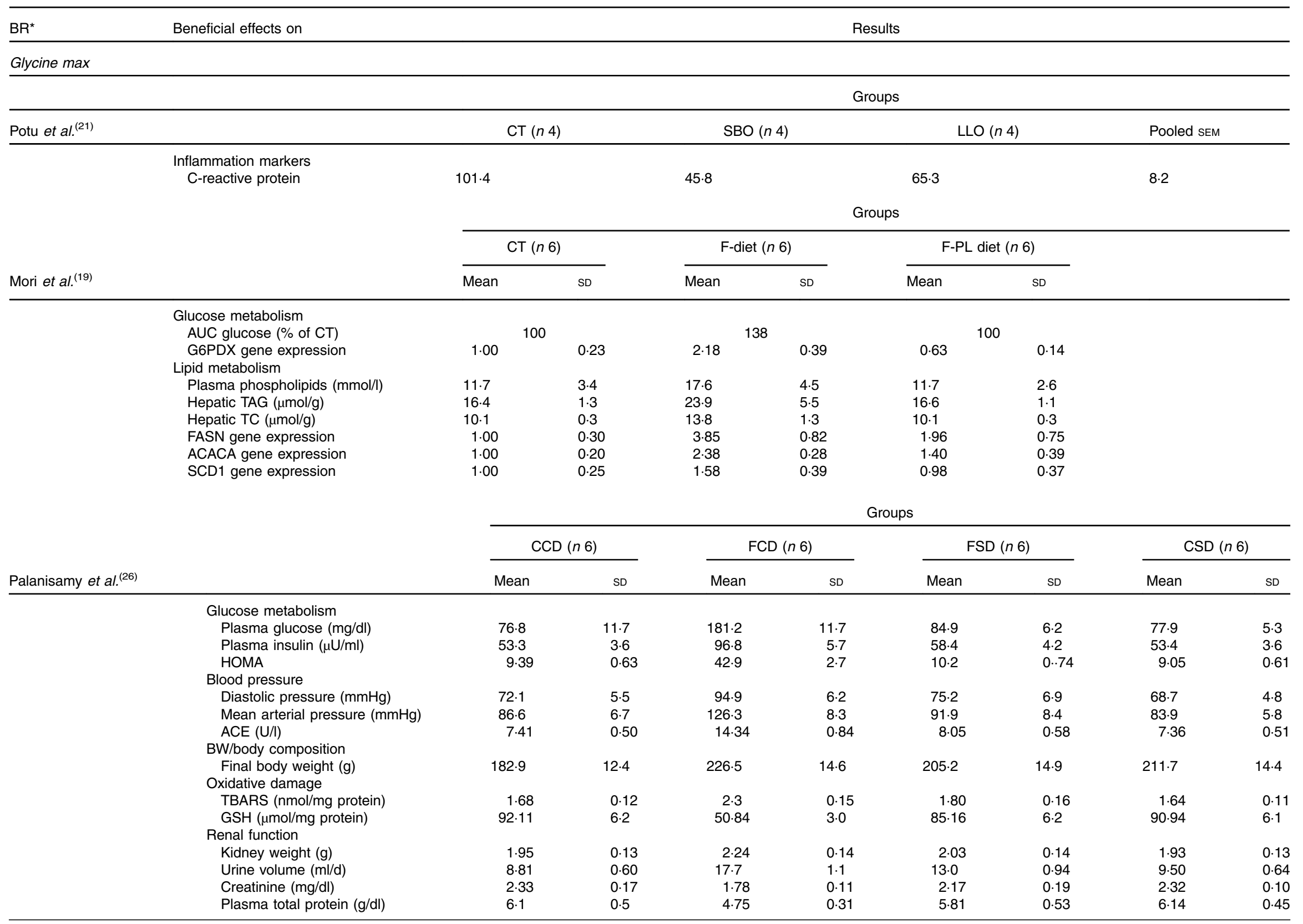




\section{N British Journal of Nutrition}

Table 2. Continued

\begin{tabular}{|c|c|c|c|c|c|c|c|}
\hline \multirow[b]{3}{*}{ Ronis et al. ${ }^{(17)}$} & \multirow[b]{3}{*}{ Expt 1} & \multicolumn{6}{|c|}{ Groups } \\
\hline & & \multicolumn{2}{|c|}{ CAS $(n 7-10)$} & \multicolumn{2}{|c|}{$\mathrm{SPI}+(n 7-10)$} & \multicolumn{2}{|c|}{ SPI- $(n 7-10)$} \\
\hline & & Mean & SEM & Mean & SEM & Mean & SEM \\
\hline & \multirow{2}{*}{\multicolumn{7}{|c|}{$\begin{array}{l}\text { Glucose metabolism } \\
\text { Glucokinase gene expression }\end{array}$}} \\
\hline & & & & & & & \\
\hline & Male & 1.00 & 0.24 & $6 \cdot 10$ & 1.90 & 0.94 & 0.12 \\
\hline & \multicolumn{7}{|l|}{ Lipid metabolism } \\
\hline & \multicolumn{7}{|l|}{ ACO gene expression } \\
\hline & Male & 1.00 & 0.06 & 1.86 & 0.08 & 1.12 & 0.09 \\
\hline & Female & 1.03 & 0.13 & 2.93 & $0 \cdot 12$ & $1 \cdot 16$ & 0.18 \\
\hline & \multicolumn{7}{|l|}{ CPT-1A expression } \\
\hline & Male & 1.00 & 0.15 & 1.74 & 0.19 & 0.99 & 0.15 \\
\hline & Female & 0.42 & 0.05 & 3.55 & 0.30 & 1.28 & 0.37 \\
\hline & \multicolumn{7}{|l|}{ HADHA expression } \\
\hline & Male & 1.00 & 0.12 & 1.61 & 0.19 & $1 \cdot 10$ & 0.07 \\
\hline & Female & 0.81 & 0.04 & 1.46 & 0.11 & $2 \cdot 10$ & 0.40 \\
\hline & \multicolumn{7}{|l|}{ PPAR $a$ expression } \\
\hline & Male & 1.00 & 0.08 & 1.50 & 0.06 & & \\
\hline & Female & 0.66 & 0.05 & $1 \cdot 18$ & 0.05 & & \\
\hline & \multicolumn{7}{|l|}{ PPAR $y$ expression } \\
\hline & Male & 1.00 & 0.06 & $2 \cdot 20$ & 0.28 & & \\
\hline & Female & 1.04 & 0.08 & 1.87 & 0.13 & & \\
\hline & \multicolumn{7}{|l|}{ CYP/A-1 gene expression } \\
\hline & Male & 1.00 & 0.19 & 3.12 & 0.47 & 4.60 & 0.60 \\
\hline & Female & 1.45 & 0.28 & 3.84 & 0.29 & 2.58 & 0.56 \\
\hline & \multicolumn{7}{|l|}{ ABCG5 gene expression } \\
\hline & Male & 1.00 & 0.16 & 1.45 & 0.06 & 0.63 & 0.13 \\
\hline & Female & 0.41 & 0.06 & $2 \cdot 17$ & 0.28 & 0.80 & 0.07 \\
\hline & \multicolumn{7}{|l|}{ ABCG8 gene expression } \\
\hline & Male & 1.00 & 0.17 & 2.85 & 0.42 & 3.34 & 0.26 \\
\hline & Female & $1 \cdot 25$ & 0.17 & 4.78 & 0.86 & 1.90 & 0.30 \\
\hline & \multicolumn{7}{|l|}{$\mathrm{LXR} a$} \\
\hline & Male & 1.00 & 0.04 & 1.38 & 0.06 & & \\
\hline & \multirow[t]{3}{*}{ Female } & 1.42 & 0.06 & 1.48 & 0.06 & & \\
\hline & & \multicolumn{6}{|c|}{ Groups } \\
\hline & & \multicolumn{2}{|c|}{ Casein $(n 7-10)$} & \multicolumn{2}{|c|}{ Wester casein $(n$ 7-10) } & \multicolumn{2}{|c|}{ Wester SPI ( $n$ 7-10) } \\
\hline & Expt 2 & Mean & SEM & Mean & SEM & Mean & SEM \\
\hline & Glucose metabolism & & & & & & \\
\hline & Serum glucose $(\mathrm{mmol} / \mathrm{l})$ & 4.50 & 0.13 & $5 \cdot 10$ & 0.17 & 4.50 & 0.21 \\
\hline & Lipid metabolism & & & & & & \\
\hline & Serum TC $(\mathrm{mmol} / \mathrm{l})$ & $2 \cdot 10$ & 0.33 & 4.40 & 1.00 & $2 \cdot 60$ & 0.36 \\
\hline & Liver weight (g/100 g body weight) & $4 \cdot 10$ & $0 \cdot 12$ & 4.70 & 0.17 & 4.20 & 0.14 \\
\hline & Liver TAG ( $\mu \mathrm{mol} / \mathrm{g}$ wet tissue) & $44 \cdot 70$ & $12 \cdot 70$ & $100 \cdot 60$ & 14.40 & $51 \cdot 20$ & $11 \cdot 30$ \\
\hline & BW/body composition & & & & & & \\
\hline & Body weight gain (g/d) & $8 \cdot 10$ & 0.10 & $9 \cdot 20$ & 0.10 & $8 \cdot 30$ & 0.20 \\
\hline & Body fat mass (\%) & $14 \cdot 80$ & 0.80 & $19 \cdot 00$ & 0.30 & $16 \cdot 00$ & $1 \cdot 20$ \\
\hline
\end{tabular}




\section{Writish Journal of Nutrition}

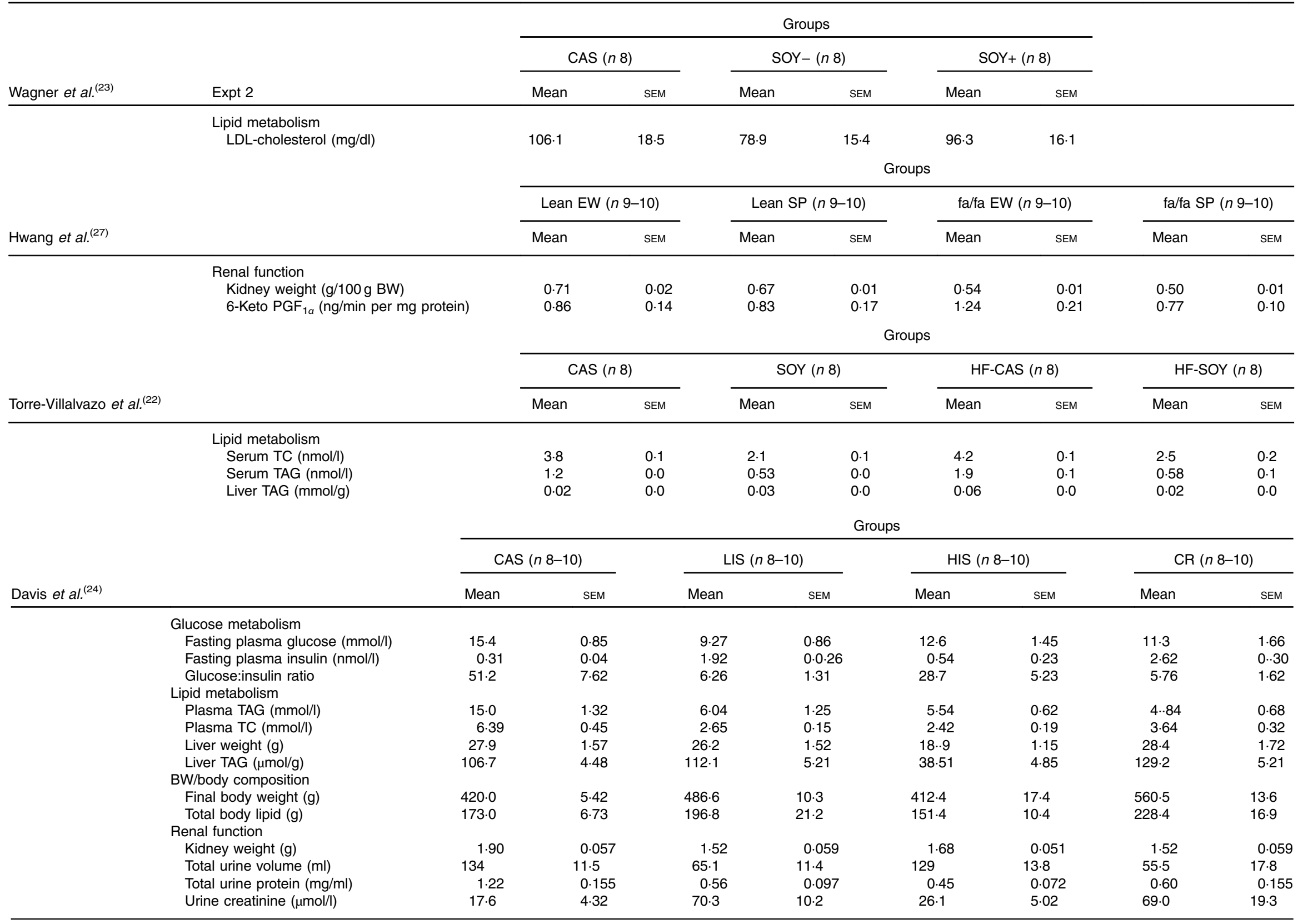




\section{N British Journal of Nutrition}

\begin{tabular}{|c|c|c|c|c|c|c|c|c|c|c|c|c|c|}
\hline \multirow[b]{3}{*}{ Barrios-Ramos et al. ${ }^{(18)}$} & & \multicolumn{12}{|c|}{ Groups } \\
\hline & & \multicolumn{2}{|c|}{$-\mathrm{CT}(n 8)$} & \multicolumn{2}{|c|}{$+\mathrm{CT}(n 8)$} & \multicolumn{2}{|c|}{ Co $(n 8)$} & \multicolumn{2}{|c|}{$\mathrm{S}(n 8)$} & \multicolumn{2}{|c|}{$\mathrm{O}(n 8)$} & \multicolumn{2}{|c|}{$\Omega(n 8)$} \\
\hline & & Mean & SEM & Mean & SEM & Mean & SEM & Mean & SEM & Mean & SEM & Mean & SEM \\
\hline & \multirow[t]{3}{*}{$\begin{array}{l}\text { Glucose metabolism } \\
\text { Serum glucose (mg/dl) }\end{array}$} & $96 \cdot 4$ & $10 \cdot 3$ & $124 \cdot 8$ & $6 \cdot 3$ & 84.6 & $7 \cdot 2$ & & & & & 91.8 & $9 \cdot 1$ \\
\hline & & \multicolumn{2}{|c|}{$\mathrm{Co}+\mathrm{S}(n 8)$} & \multicolumn{2}{|c|}{$\mathrm{Co}+\mathrm{O}(n 8)$} & \multicolumn{2}{|c|}{$\mathrm{Co}+\Omega(n 8)$} & \multicolumn{2}{|c|}{$\operatorname{coso} \Omega(n 8)$} & \multicolumn{2}{|c|}{$\operatorname{CoSO} \Omega$ ASM $(n 8)$} & \multicolumn{2}{|c|}{$\operatorname{CoSO} \Omega$ BSM $(n 8)$} \\
\hline & & \multicolumn{2}{|c|}{$\begin{array}{c}- \\
-\mathrm{CT}(n 8)\end{array}$} & \multicolumn{2}{|c|}{$\begin{array}{c}- \\
+\mathrm{CT}(n 8)\end{array}$} & \multicolumn{2}{|c|}{$\begin{array}{c}- \\
\text { Co }(n 8)\end{array}$} & \multicolumn{2}{|c|}{$\begin{array}{c}- \\
S(n 8)\end{array}$} & $\mathrm{O}(n 8)$ & $3 \cdot 7$ & \multicolumn{2}{|c|}{$\begin{array}{c}- \\
\Omega(n 8)\end{array}$} \\
\hline & \multirow{3}{*}{$\begin{array}{l}\text { Lipid metabolism } \\
\text { Total cholesterol (mmol/l) }\end{array}$} & 14.2 & 0.95 & $86 \cdot 4$ & $12 \cdot 0$ & \multirow{2}{*}{\multicolumn{2}{|c|}{$\begin{array}{c}- \\
\mathrm{Co}+{ }^{\prime} \Omega(n 8)\end{array}$}} & $68 \cdot 2$ & 7.7 & \multirow{2}{*}{\multicolumn{2}{|c|}{$\operatorname{CoSO}^{-} \overline{\mathrm{ASM}}(n 8)$}} & & \\
\hline & & \multicolumn{2}{|c|}{$\mathrm{Co}+\mathrm{S}(n 8)$} & \multicolumn{2}{|c|}{$\mathrm{Co}+\mathrm{O}(n 8)$} & & & \multicolumn{2}{|c|}{$\operatorname{Coso} \Omega(n 8)$} & & & Coso's & $(n 8)$ \\
\hline & & & & & & & & & & & & & \\
\hline & HDL-cholesterol $(\mathrm{mmol} / \mathrm{l})$ & & & $+\mathrm{C}$ & & Co & & & & & & & \\
\hline & & & & $6 \cdot 7$ & 0.64 & 4.5 & 0.16 & 3.1 & 0.72 & 4.0 & 0.52 & 3.9 & 0.25 \\
\hline & & $\mathrm{Co}+\mathrm{r}-\mathrm{r}-\mathrm{r}$ & (n 8) & $\mathrm{Co}+$ & n 8) & $\mathrm{Co}+$ & n 8) & $\cos$ & n 8) & Coso's & $(n 8)$ & Coso's & $(n 8)$ \\
\hline & & & 0.25 & & & & 0.40 & & & & & 3.5 & 0.36 \\
\hline & LDL-cholesterol (mmol/l) & & & $+\mathrm{C}$ & & Co & & & & & & & \\
\hline & & & 1.01 & $79 \cdot 0$ & $12 \cdot 3$ & & & & & & & & \\
\hline & & $\mathrm{Co}+$ & (n 8) & $\mathrm{Co}+$ & n 8) & $\mathrm{Co}+$ & n 8) & $\cos$ & n 8) & CosO's & $1(n 8)$ & Coso: & $(n 8)$ \\
\hline & & & & & & & & & & & & & \\
\hline & & & & $+\mathrm{C}^{-}$ & & Co & & & & & & & \\
\hline & TAG $(\mathrm{mmol} / \mathrm{l})$ & 0.68 & 0.05 & 1.54 & 0.11 & 0.57 & 0.06 & 0.87 & 0.09 & 0.73 & 0.11 & 0.68 & 0.08 \\
\hline & & $\mathrm{Co}+$ & (n 8) & $\mathrm{Co}+$ & n 8) & $\mathrm{Co}+$ & n 8) & $\cos$ & n 8) & Coso's & $(n 8)$ & Coso & $(n 8)$ \\
\hline & & 0.80 & 0.09 & 0.80 & 0.05 & 0.90 & 0.16 & 0.82 & 0.07 & 0.92 & 0.04 & 1.18 & 0.09 \\
\hline & & & & $+\mathrm{C}$ & & Co & & & & & & & \\
\hline & Steatosis (\%) & & & 29.9 & 1.32 & & & & 1.09 & & & & \\
\hline & & $\mathrm{Co}+$ & (n 8) & $\mathrm{Co}+$ & n 8) & $\mathrm{Co}+$ & (n 8) & $\cos$ & n 8) & Coso's & $1(n 8)$ & Coso: & $(n 8)$ \\
\hline & & 4.71 & 0.69 & $6 \cdot 29$ & 0.74 & 21.08 & 1.64 & 55.04 & 1.34 & $10 \cdot 11$ & 0.68 & & \\
\hline Trigonella foenum graec & fenugreek & & & & & & & & & & & & \\
\hline & & & & & & & & & & & & & \\
\hline & & & & $\mathrm{HF}$ & & Fen & & & & & & & \\
\hline Muraki et al. ${ }^{(28)}$ & & Mean & SEM & Mean & SEM & Mean & SEM & & & & & & \\
\hline & Glucose metabolism & & & & & & & & & & & & \\
\hline & HOMA-IR & 1.00 & 0.34 & $2 \cdot 30$ & 0.31 & 1.32 & 0.24 & & & & & & \\
\hline
\end{tabular}


Bs British Journal of Nutrition

Table 2. Continued

Groups

\begin{tabular}{|c|c|c|c|c|c|c|c|c|c|c|c|c|c|c|c|c|c|c|}
\hline \multirow[b]{3}{*}{ Kannappan \& Anuradha ${ }^{(33)}$} & & & & & \multirow{2}{*}{\multicolumn{2}{|c|}{$\operatorname{CON}(n 6)$}} & & & & & & & & & & & & \\
\hline & & & & & & & \multicolumn{3}{|c|}{$\mathrm{FRU}(n 6)$} & \multicolumn{3}{|c|}{$\mathrm{FRU}+$ FPEt $(n 6)$} & \multicolumn{3}{|c|}{$\mathrm{FRU}+$ Quer $(n 6)$} & \multicolumn{3}{|c|}{$\mathrm{FRU}+\operatorname{Met}(n 6)$} \\
\hline & & & & & Mean & SD & & Mean & SD & & lean & SD & & lean & SD & $\mathrm{Me}$ & ean & SD \\
\hline & \multicolumn{18}{|c|}{ Glucose metabolism } \\
\hline & \multirow{2}{*}{\multicolumn{4}{|c|}{$\begin{array}{l}\text { Plasma glucose }(\mathrm{mm}) \\
\text { Plasma insulin }(\mu \mathrm{U} / \mathrm{ml})\end{array}$}} & 4.51 & 0.21 & & $7 \cdot 15$ & 0.15 & & $5 \cdot 66$ & 0.21 & & $5 \cdot 22$ & 0.37 & & 4.69 & 0.27 \\
\hline & & & & & 46.58 & 3.87 & & $83 \cdot 10$ & $6 \cdot 37$ & & 65.38 & 3.88 & & 60.03 & 4.60 & & 50.06 & $4 \cdot 20$ \\
\hline & \multicolumn{4}{|c|}{ HOMA } & 9.32 & 0.76 & & $26 \cdot 44$ & 1.39 & & $15 \cdot 63$ & 0.96 & & 13.23 & 0.83 & & 10.48 & 0.81 \\
\hline & \multicolumn{4}{|c|}{ QUICKY } & 0.283 & 0.017 & & 0.248 & 0.019 & & 0.257 & 0.012 & & 0.267 & 0.021 & & 0.275 & 0.019 \\
\hline & \multicolumn{4}{|c|}{$I S I_{0,120}$} & 129.03 & 9.87 & & $60 \cdot 87$ & 3.56 & & 84.06 & 4.29 & & $05 \cdot 11$ & $5 \cdot 88$ & & 21.47 & 7.35 \\
\hline & \multicolumn{4}{|c|}{ AUC glucose $(\mathrm{mg} / \mathrm{ml}$ per $\mathrm{min})$} & 159.5 & 11.23 & & 271.94 & 21.60 & & 03.47 & $12 \cdot 45$ & & 93.5 & $11 \cdot 30$ & & $75 \cdot 70$ & $9 \cdot 30$ \\
\hline & \multicolumn{4}{|c|}{ AUC insulin ( $\mathrm{mg} / \mathrm{ml}$ per min) } & 10021 & 823 & 1665 & 552 & 1060 & 1299 & & 993 & 1190 & & 1030 & 1064 & 49 & 956 \\
\hline & \multicolumn{4}{|c|}{ Hexokinase $\dagger$} & 0.839 & 0.02 & & 0.392 & 0.01 & & 0.656 & 0.0010 & & 0.701 & 0.009 & & 0.815 & 0.04 \\
\hline & \multicolumn{4}{|c|}{ Pyruvate kinase } & $113 \cdot 27$ & 6.53 & & 69.83 & 4.39 & & $80 \cdot 88$ & 5.05 & & 92.06 & 8.42 & & $06 \cdot 32$ & 8.27 \\
\hline & \multicolumn{4}{|c|}{ G6Pase§ } & $4 \cdot 21$ & 0.24 & & 7.94 & 0.21 & & $6 \cdot 31$ & 0.22 & & $5 \cdot 32$ & 0.25 & & 4.53 & 0.34 \\
\hline & \multicolumn{4}{|c|}{ F1,6BPase§ } & 4.75 & 0.19 & & 8.84 & 0.52 & & $6 \cdot 18$ & 0.29 & & 5.60 & 0.24 & & 5.02 & 0.21 \\
\hline & \multicolumn{4}{|c|}{ GP\| } & $4 \cdot 11$ & 0.21 & & 7.44 & 0.30 & & 6.43 & 0.29 & & $5 \cdot 47$ & 0.45 & & 4.40 & 0.27 \\
\hline & \multicolumn{4}{|c|}{ Glycogen (mg Glu/g tissue) } & 54.78 & $5 \cdot 21$ & & $32 \cdot 14$ & $3 \cdot 12$ & & 39.91 & $3 . .85$ & & $45 \cdot 54$ & 3.93 & & 51.59 & 5.00 \\
\hline & ICDHII & & & & 741.2 & 28.8 & & 538.8 & $26 \cdot 1$ & & 28.5 & 20.5 & & 68.5 & 29.8 & & $10 \cdot 3$ & $15 \cdot 6$ \\
\hline & $\mathrm{SDH}(\mathrm{m}$ & ng glucos & e/g tissue) & & $28 \cdot 74$ & 2.56 & & 11.49 & 0.96 & & $15 \cdot 74$ & 0.99 & & 18.91 & 1.58 & & $26 \cdot 23$ & 1.94 \\
\hline & PTP $\left(A_{E}\right.$ & $620)$ & & & 0.458 & 0.02 & & 0.731 & 0.04 & & 0.595 & 0.03 & & 0.567 & 0.02 & & 0.475 & 0.02 \\
\hline & PTK (A & 492) & & & 0.672 & 0.04 & & 0.335 & 0.01 & & 0.597 & 0.04 & & 0.637 & 0.03 & & 0.659 & 0.02 \\
\hline & Lipid meta & abolism & & & & & & & & & & & & & & & & \\
\hline & Plasma & TAG & $g / d l)$ & & 89.01 & $4 \cdot 20$ & & 163.42 & $5 \cdot 39$ & & $28 \cdot 40$ & 6.08 & & $17 \cdot 47$ & 8.63 & & $94 \cdot 30$ & 3.34 \\
\hline & Plasma & NEFA ( & $\mathrm{ng} / \mathrm{dl}$ ) & & $25 \cdot 68$ & $2 \cdot 42$ & & $72 \cdot 24$ & 6.35 & & $58 \cdot 12$ & 4.69 & & 47.99 & 2.56 & & $31 \cdot 17$ & $2 \cdot 67$ \\
\hline & & & & & & & & & Group & & & & & & & & & \\
\hline & CT ( & & $0.5 \mathrm{FS}$ & $P(n 5)$ & $1.0 \mathrm{FSF}$ & $\mathrm{P}(n 5)$ & Allx ( & $(n 5)$ & $\begin{array}{r}\text { Alloxan }+C \\
(n 5\end{array}$ & $\begin{array}{l}0.5 \mathrm{FSP} \\
5)\end{array}$ & Allx $+1 F S$ & SP $(n 5)$ & $\mathrm{CHOL}$ & $L(n 5)$ & $\mathrm{CHOL}+0.5$ & $\operatorname{FSP}(n 5)$ & $\begin{array}{r}\mathrm{CHOL}+1 \\
(n 5\end{array}$ & $\begin{array}{l}\text { 1.0 FSP } \\
\text { 5) }\end{array}$ \\
\hline Ramadan et al. ${ }^{(29)}$ & Mean & SEM & Mean & SEM & Mean & SEM & Mean & SEM & Mean & SEM & Mean & SEM & Mean & SEM & Mean & SEM & Mean & SEM \\
\hline Glucose metabolism & & & & & & & & & & & & & & & & & & \\
\hline $\begin{array}{c}\text { Serum glucose } \\
(\mathrm{mg} / \mathrm{l})\end{array}$ & 930 & 45 & 834 & 9 & 751 & 4 & 3028 & 52 & 1847 & 12 & 1163 & 16 & 1512 & 21 & 1177 & 12 & 1033 & 12 \\
\hline Lipid metabolism & & & & & & & & & & & & & & & & & & \\
\hline $\begin{array}{l}\text { Liver weight:BW } \\
\text { ratio }\end{array}$ & 0.0304 & 0.0010 & 0.0322 & 0.0004 & 0.0326 & 0.0010 & 0.0327 & 0.0002 & 0.0315 & 0.0003 & 0.0310 & 0.0001 & 0.0422 & 0.0007 & 0.0398 & 0.0003 & 0.0380 & 0.0003 \\
\hline $\begin{array}{l}\text { ratio } \\
\text { Serum total lipids } \\
(\mathrm{mg} / \mathrm{l})\end{array}$ & 4614 & 178 & 4500 & 148 & 3592 & 125 & 7047 & 141 & 6319 & 126 & 5485 & 75 & 8064 & 102 & 5943 & 118 & 5479 & 99 \\
\hline TC $(\mathrm{mg} / \mathrm{l})$ & 586 & 21 & 534 & 10 & 519 & 4 & 855 & 26 & 748 & 8 & 705 & 7 & 1403 & 14 & 908 & 10 & 819 & 11 \\
\hline TAG $(\mathrm{mg} / \mathrm{l})$ & 522 & 6 & 444 & 12 & $\begin{array}{r}345 \\
\end{array}$ & 4 & 1130 & 23 & 975 & & 754 & 6 & 1110 & 18 & 935 & & 686 & 13 \\
\hline $\begin{array}{l}\text { Atherogenic indexף } \\
\text { Atherogenic index } x^{\star}\end{array}$ & 1409 & 0.022 & 1241 & 0.007 & 1177 & 0.002 & 3478 & 0.086 & $\begin{array}{l}2924 \\
1161\end{array}$ & 0.086 & 2084 & 0.028 & 4272 & $\begin{array}{l}0.079 \\
0.071\end{array}$ & 2665 & 0.026 & 1991 & $\begin{array}{l}0.010 \\
0.010\end{array}$ \\
\hline $\begin{array}{l}\text { Atherogenic index* } \\
\text { BW/body composition }\end{array}$ & 0.157 & 0.018 & 0.034 & 0.007 & 0.020 & 0.002 & 1557 & 0.055 & 1161 & 0.046 & 0.638 & 0.022 & 2596 & 0.071 & 1116 & 0.023 & 0.657 & 0.010 \\
\hline $\begin{array}{l}\text { BW/body composition } \\
\text { Body weight gain or loss (g) }\end{array}$ & 37.4 & 0.7 & 39.0 & 0.3 & 39.6 & 0.7 & $-14 \cdot 2$ & 0.6 & 7.4 & 0.4 & 20.0 & 0.8 & 78.4 & 0.9 & 65.6 & 0.7 & $51 \cdot 4$ & $1 \cdot 1$ \\
\hline $\begin{array}{l}\text { Inflammation markers } \\
\text { ALAT activity (IU/I) }\end{array}$ & & 1.1 & 27.5 & 0.6 & $24 \cdot 3$ & 0.8 & 90.0 & 1.0 & 69.2 & 0.8 & 37.6 & 0.5 & 91.7 & 3.2 & 66.8 & 1.0 & 39.3 & 1.1 \\
\hline ASAT activity (IU/I) & 39.2 & 2.2 & 36.1 & 0.8 & 34.4 & 1.1 & 148.6 & 1.7 & 84.8 & 1.1 & 63.2 & 1.2 & 126.6 & 2.1 & 65.1 & 1.5 & 44.0 & 1.2 \\
\hline ALP activity (IU/I) & 29.0 & 1.4 & 27.7 & 1.2 & $24 \cdot 6$ & 0.7 & 101.0 & $2 \cdot 3$ & 59.4 & 1.8 & $41 \cdot 2$ & 1.1 & 45.1 & 0.5 & 39.5 & 0.9 & 37.4 & 0.8 \\
\hline $\begin{array}{l}\text { Oxidative damage } \\
\text { GSH (nm/g tissue) }\end{array}$ & 10.2 & 0.3 & $12 \cdot 1$ & 0.2 & 13.5 & 0.4 & 2.5 & 0.1 & 3.7 & 0.2 & 4.4 & 0.1 & 8.9 & 0.1 & 10.5 & 0.2 & 11.3 & 0.3 \\
\hline
\end{tabular}




\section{Nesitish Journal of Nutrition}

Table 2. Continued

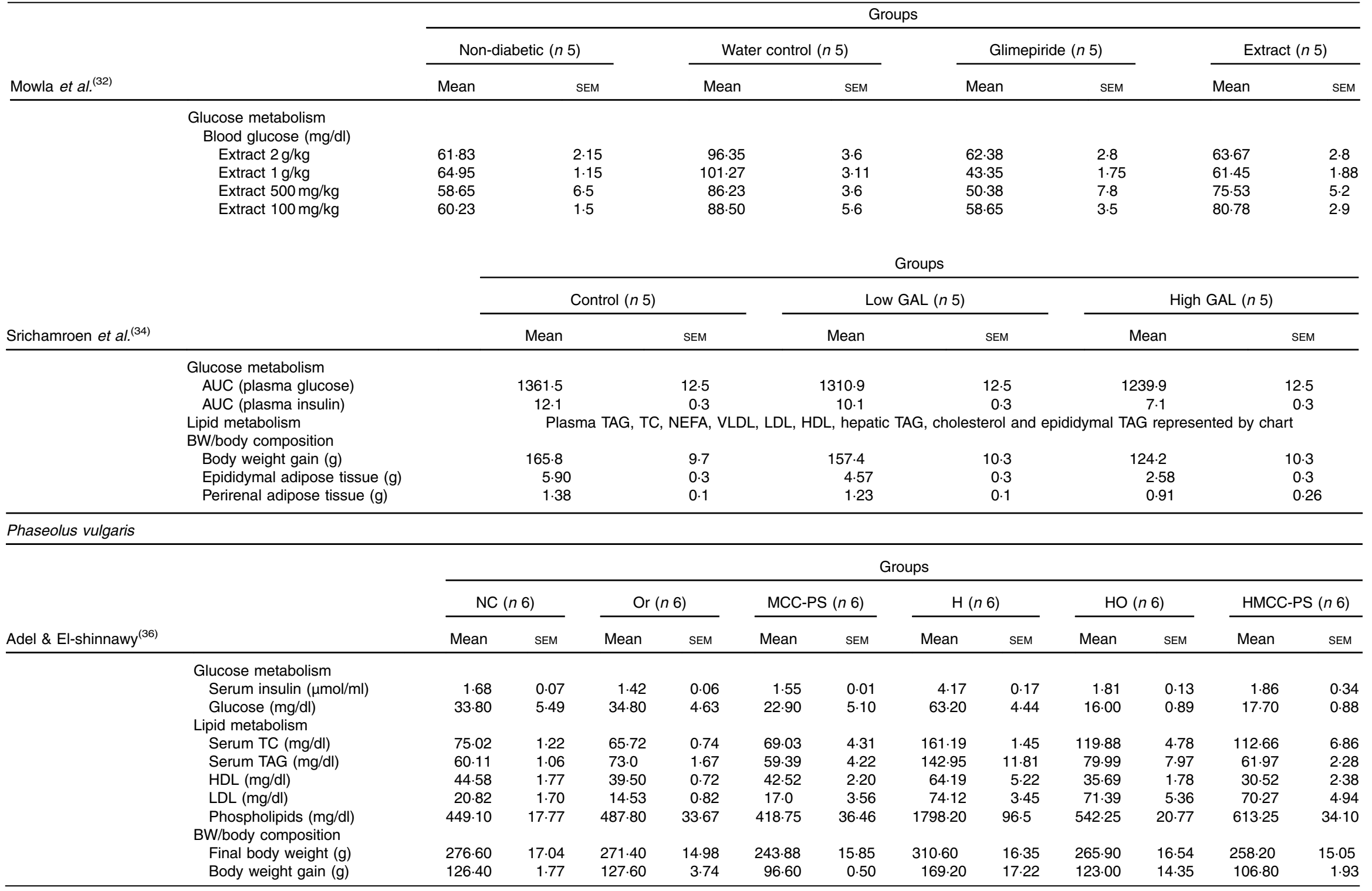




\section{N British Journal of Nutrition}

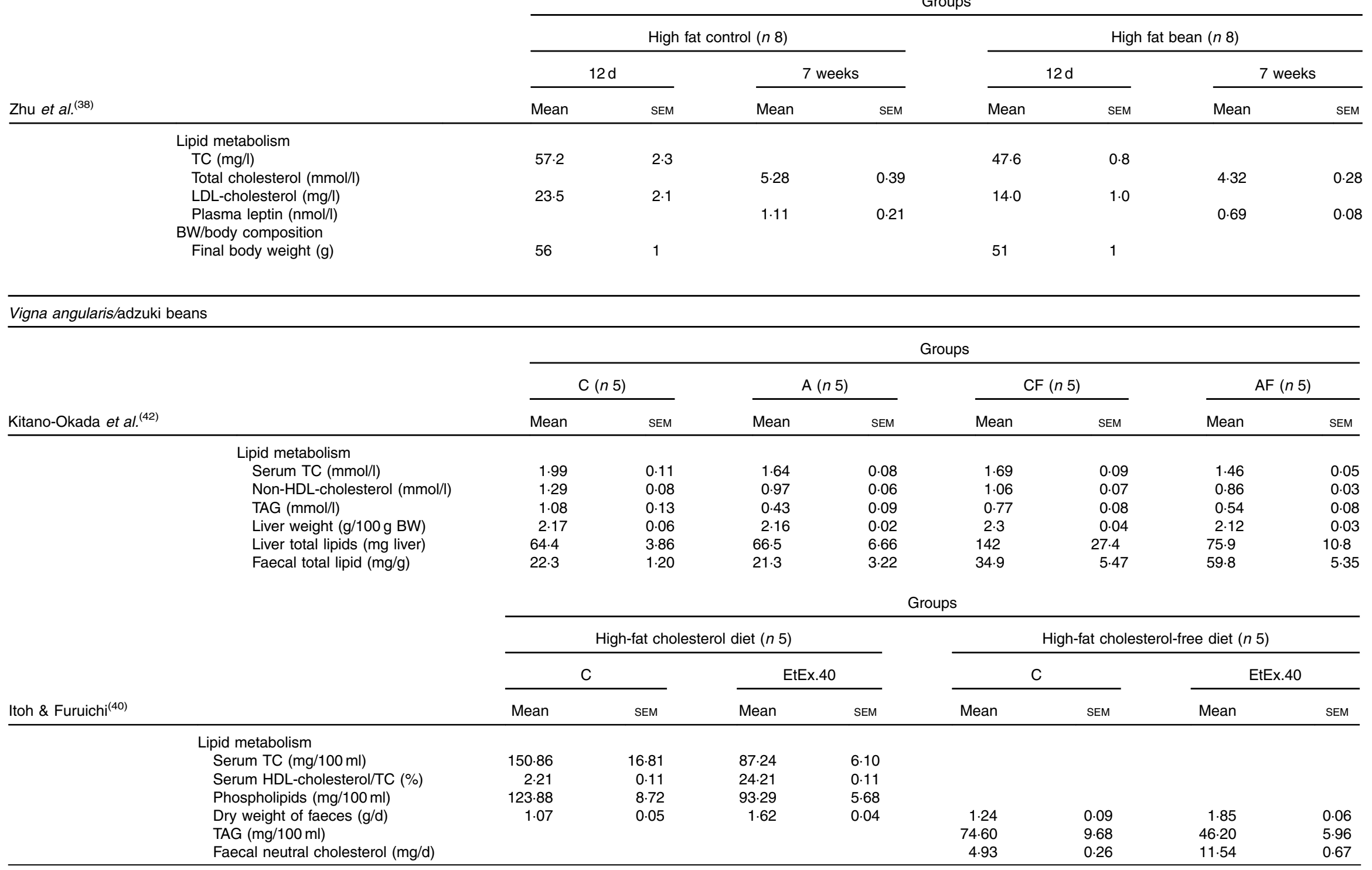




\section{N British Journal of Nutrition}

Table 2. Continued

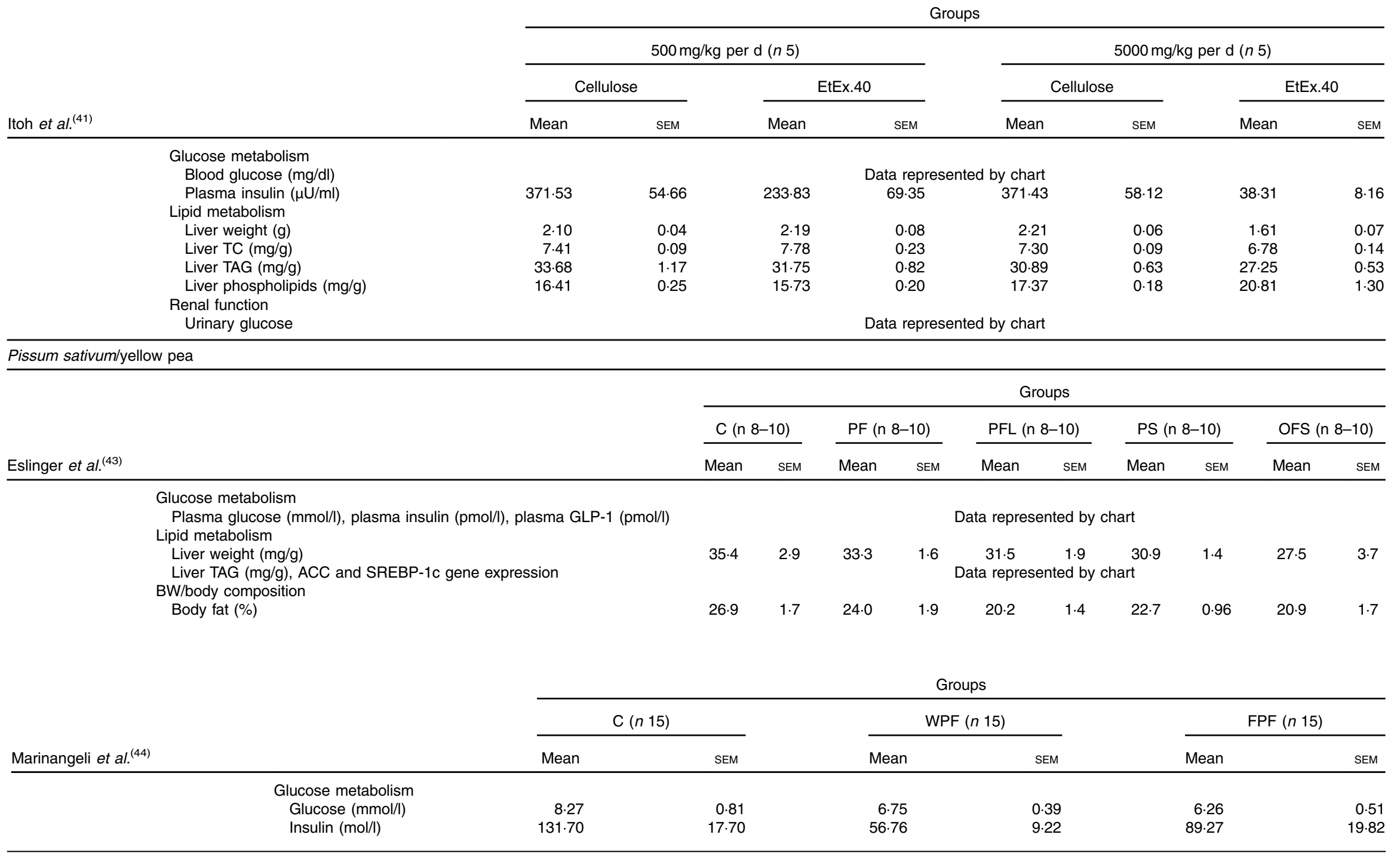




\section{W British Journal of Nutrition}

Table 2. Continued

Glycyrrhiza glabra

\begin{tabular}{|c|c|c|c|c|c|c|c|c|c|}
\hline \multirow[b]{3}{*}{ Aoki et al. ${ }^{(4)}$} & & \multicolumn{8}{|c|}{ Groups } \\
\hline & & \multicolumn{2}{|c|}{ CT (n 10) } & \multicolumn{2}{|c|}{$0.5 \%$ LFO (n 10) } & \multicolumn{2}{|c|}{$1 \%$ LFO (n 10) } & \multicolumn{2}{|c|}{$2 \%$ LFO (n 10) } \\
\hline & & Mean & SD & Mean & SD & Mean & SD & Mean & SD \\
\hline & $\begin{array}{l}\text { Glucose metabolism } \\
\text { Serum insulin (ng/ml) } \\
\text { Lipids metabolism }\end{array}$ & 1.69 & 0.88 & 1.73 & 1.23 & $0 \cdot 80$ & 0.50 & 0.70 & 0.42 \\
\hline & $\begin{array}{l}\text { Serum leptin }(\mathrm{ng} / \mathrm{ml}) \\
\text { Adipose mesenteric weight }(\mathrm{g}) \\
\text { Adipose periuterine weight }(\mathrm{g}) \\
\text { Adipose perirenal weight }(\mathrm{g}) \\
\text { BW/body composition }\end{array}$ & $\begin{array}{c}35 \cdot 3 \\
0.611 \\
1500 \\
0.958\end{array}$ & $\begin{array}{l}12 \cdot 3 \\
0 \cdot 160 \\
0 \cdot 415 \\
0 \cdot 248\end{array}$ & $\begin{array}{c}35.4 \\
0.572 \\
1444 \\
0.894\end{array}$ & $\begin{array}{c}13.5 \\
0.189 \\
0.465 \\
0.297\end{array}$ & $\begin{array}{c}17 \cdot 0 \\
0.419 \\
0.980 \\
0.589\end{array}$ & $\begin{array}{l}7 \cdot 6 \\
0 \cdot 117 \\
0.360 \\
0.230\end{array}$ & $\begin{array}{l}6.54 \\
0.284 \\
0.541 \\
0.324\end{array}$ & $\begin{array}{l}6 \cdot 04 \\
0 \cdot 115 \\
0.238 \\
0 \cdot 187\end{array}$ \\
\hline & Body weight gain (g/8 week) & $6 \cdot 2$ & $2 \cdot 2$ & 5.9 & 1.7 & $2 \cdot 4$ & $1 \cdot 8$ & 0.3 & 1.9 \\
\hline
\end{tabular}

Cajanus cajan

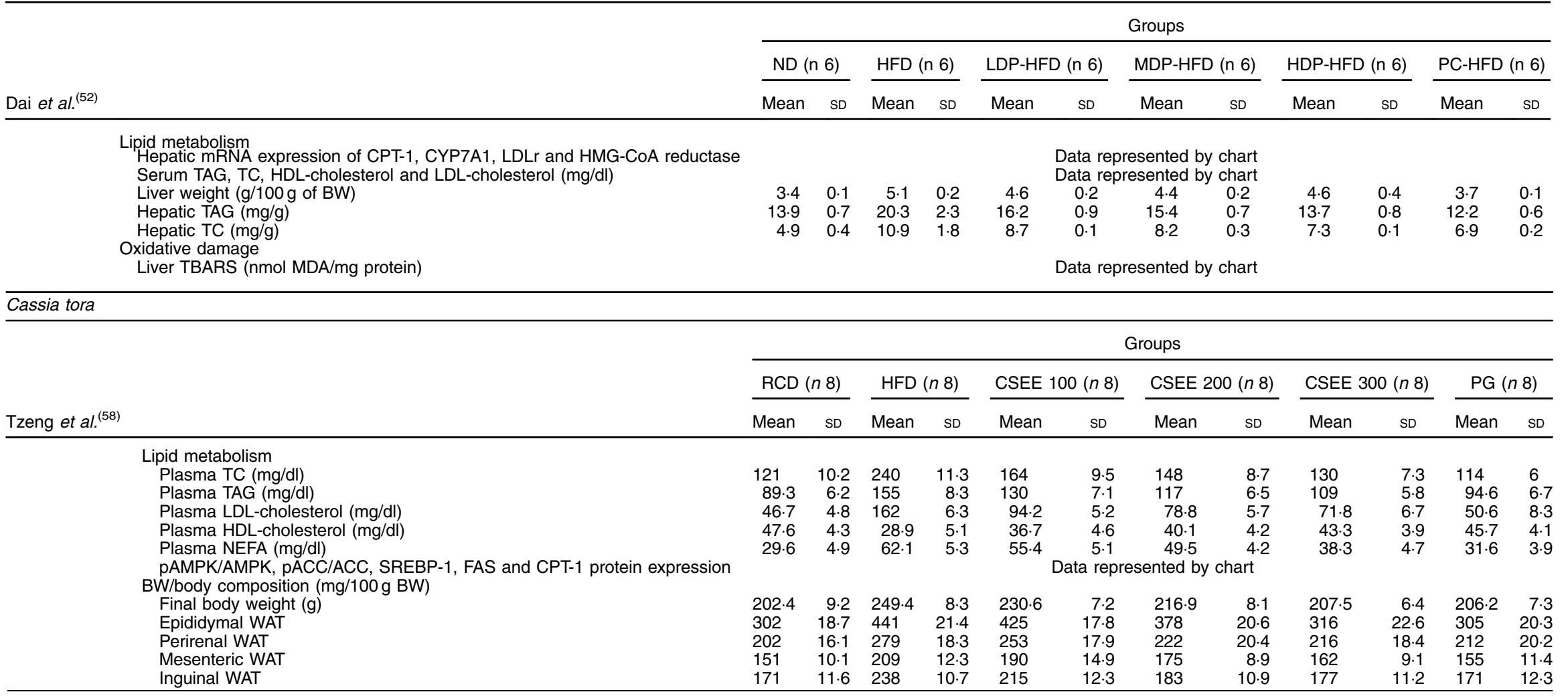




\section{Nesitish Journal of Nutrition}

Table 2. Continued

Cicer arietium/Lens culinaris

\begin{tabular}{|c|c|c|c|c|c|c|c|}
\hline \multirow[b]{3}{*}{ Boualga et al. ${ }^{(61)}$} & & \multicolumn{6}{|c|}{ Groups } \\
\hline & & \multicolumn{2}{|c|}{ CAS $(n 6)$} & \multicolumn{2}{|c|}{$\mathrm{CP}(n 6)$} & \multicolumn{2}{|c|}{$\mathrm{L}(n 6)$} \\
\hline & & Mean & SEM & Mean & SEM & Mean & SEM \\
\hline & \multicolumn{7}{|l|}{ Lipid metabolism } \\
\hline & Plasma TAG $(\mathrm{mmol} / \mathrm{l})$ & 0.99 & 0.23 & 0.53 & 0.13 & 0.42 & 0.19 \\
\hline & Liver TC $(\mu \mathrm{mol} / \mathrm{g})$ & $19 \cdot 31$ & $2 \cdot 27$ & 13.25 & 1.95 & 10.96 & 2.41 \\
\hline & Liver TAG $(\mu \mathrm{mol} / \mathrm{g})$ & 18.41 & 1.68 & 12.67 & 3.41 & 9.20 & 2.55 \\
\hline & Hepatic lipase and lipoprotein lipase activity & \multirow{2}{*}{\multicolumn{6}{|c|}{ Data represented by chart }} \\
\hline & BW/body composition & & & & & & \\
\hline & Body weight $(\mathrm{g})$ & 229.4 & $29 \cdot 3$ & 189.8 & 7.6 & $175 \cdot 4$ & $9 \cdot 2$ \\
\hline & Weight gain $(\mathrm{g} / \mathrm{d}$ per rat) & 5.62 & 1.40 & 3.80 & 0.90 & 3.30 & 1.01 \\
\hline & Epididymal fat weight ( $/ \mathrm{kg} \mathrm{BW})$ & $20 \cdot 19$ & $2 \cdot 70$ & $16 \cdot 70$ & 0.37 & 16.40 & 0.29 \\
\hline
\end{tabular}

Pterocarpus santaniloides

Groups

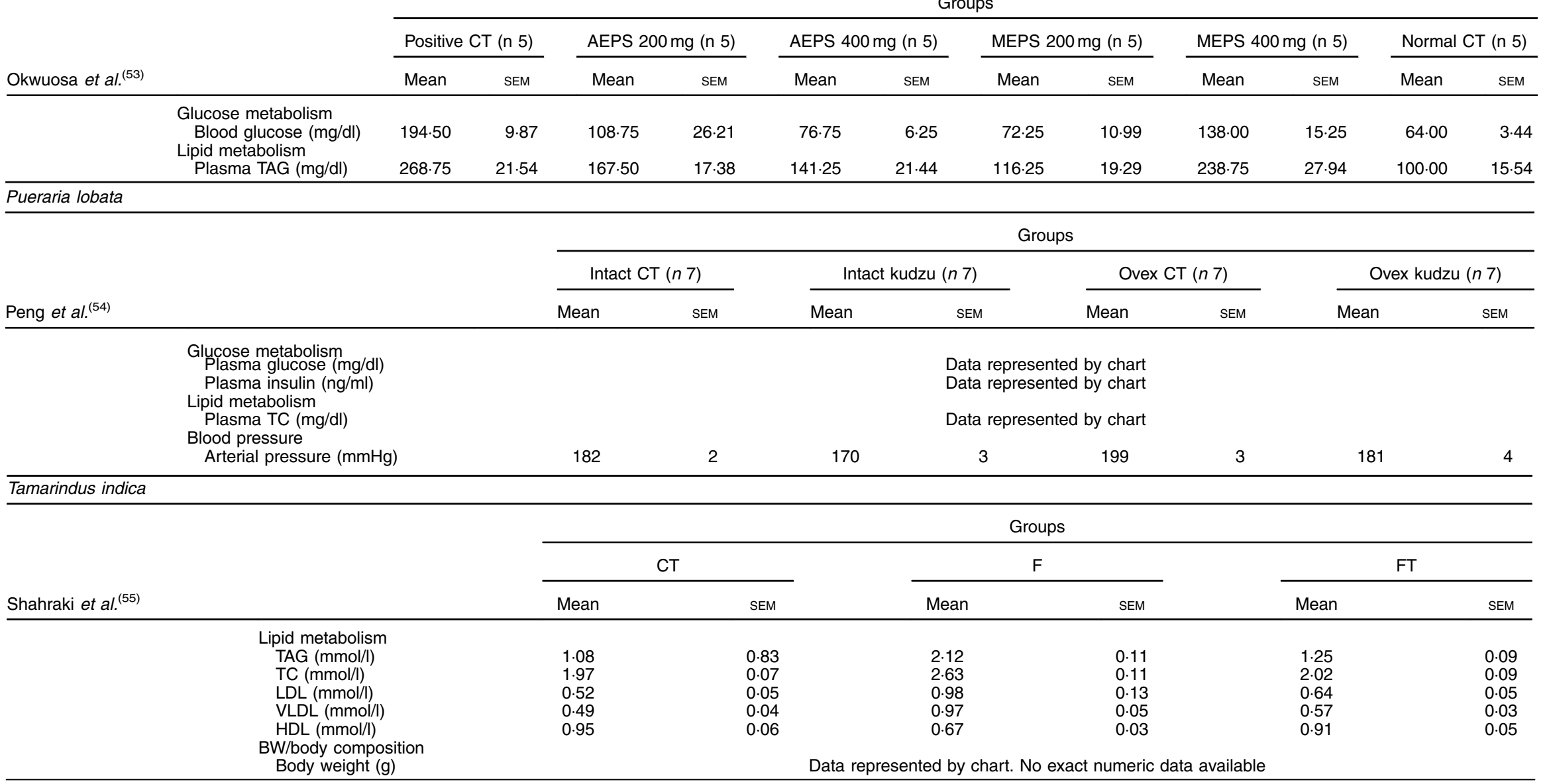


NS British Journal of Nutrition

Tephrosia purpurea

Groups

\begin{tabular}{|c|c|c|c|c|c|c|c|c|c|c|c|}
\hline \multirow[b]{2}{*}{ Pavana et al. ${ }^{(57}$} & & \multicolumn{2}{|c|}{ CT (n 6) } & \multicolumn{2}{|c|}{ Diabetic CT (n 6) } & \multicolumn{2}{|c|}{ Diabetic + TpALet (n 6) } & \multicolumn{2}{|c|}{ CT + TpALet (n 6) } & \multicolumn{2}{|c|}{ Diabetic + GLIB (n 6) } \\
\hline & & Mean & SD & Mean & SD & Mean & SD & Mean & SD & Mean & SD \\
\hline & \multicolumn{11}{|l|}{ Glucose metabolism } \\
\hline & Blood glucose (mg/dl) & 92.6 & $5 \cdot 74$ & $285 \cdot 3$ & $12 \cdot 8$ & $128 \cdot 4$ & 6.78 & 87.5 & 4.72 & $112 \cdot 6$ & $9 \cdot 2$ \\
\hline & Plasma insulin $(\mu \mathrm{U} / \mathrm{ml})$ & $16 \cdot 1$ & 0.81 & $10 \cdot 6$ & 0.87 & 14.1 & 1.03 & $16 \cdot 6$ & 0.98 & 14.7 & 1.08 \\
\hline & \multicolumn{11}{|l|}{ Lipid metabolism } \\
\hline & TC $(\mathrm{mg} / \mathrm{dl})$ & $80 \cdot 16$ & $6 \cdot 5$ & $150 \cdot 83$ & $8 \cdot 6$ & $120 \cdot 9$ & $7 \cdot 3$ & 78.7 & $5 \cdot 3$ & 114.6 & 7.5 \\
\hline & Phospholipids (mg/dl) & $93 \cdot 2$ & 6.99 & $146 \cdot 3$ & 9.83 & $116 \cdot 6$ & $8 \cdot 16$ & $91 \cdot 3$ & 7.75 & 107.5 & $9 \cdot 35$ \\
\hline & TAG $(\mathrm{mg} / \mathrm{dl})$ & 75.5 & 6.04 & 141.6 & $8 \cdot 16$ & 114.2 & 9.72 & 73.25 & 7.84 & 108.5 & $9 \cdot 35$ \\
\hline & NEFA (mg/dl) & 9.15 & 0.57 & $16 \cdot 8$ & 0.86 & $10 \cdot 6$ & 1.08 & 9.08 & 0.61 & 9.96 & 0.84 \\
\hline & HDL-cholesterol (mg/dl) & $35 \cdot 33$ & 1.47 & 21.64 & 0.73 & 28.36 & 1.08 & 36.08 & 1.35 & $29 \cdot 1$ & 0.94 \\
\hline & LDL-cholesterol (mg/dl) & 59.8 & $6 \cdot 14$ & 157.5 & 8.86 & 115.41 & 7.94 & $56 \cdot 31$ & $6 \cdot 6$ & $108 \cdot 1$ & $6 \cdot 4$ \\
\hline & VLDL-cholesterol (mg/dl) & $15 \cdot 2$ & 1.2 & $28 \cdot 3$ & 1.6 & $22 \cdot 8$ & 1.9 & $14 \cdot 6$ & 1.5 & 21.5 & $1 \cdot 8$ \\
\hline & Liver TC $(\mathrm{mg} / \mathrm{g})$ & 4.05 & 0.37 & 8.46 & 0.59 & $6 \cdot 16$ & 0.35 & 3.98 & 0.31 & $6 \cdot 2$ & 0.44 \\
\hline & Liver TAG $(\mathrm{mg} / \mathrm{g})$ & 3.89 & 0.25 & 6.54 & 0.47 & 4.96 & 0.20 & 3.86 & 0.29 & 4.61 & 0.27 \\
\hline & Liver phospholipids ( $\mathrm{mg} / \mathrm{g}$ ) & $26 \cdot 6$ & 1.7 & $42 \cdot 1$ & 2.89 & $36 \cdot 3$ & 1.63 & $19 \cdot 2$ & 1.78 & 32.83 & 1.42 \\
\hline & Liver NEFA (mg/g) & 7.58 & 1.24 & 14.5 & $2 \cdot 13$ & 11.4 & 1.31 & $7 \cdot 25$ & 0.93 & $10 \cdot 95$ & 1.39 \\
\hline
\end{tabular}

BR, bibliographic reference; CT, control diet; SBO, soyabean oil; LLO, low $a$-linolenic soyabean oil; F-diet, fructose diet; F-PL, $60 \%$ fructose diet + phospholipids from soyabeans; G6PDX, glucose-6-phosphate dehydrogenase; TC, total cholesterol; FASN, fatty acid synthase; ACACA, acetyl-Coenzyme A carboxylase alpha; SCD1, stearoyl-CoA desaturase-1; CCD, starch and casein; FCD, fructose and casein; FSD, fructose and soya protein; CSD, starch and soya protein; HOMA-IR, homoeostatic model assessment for insulin resistance; ACE, angiotensin-converting enzyme; TBARs, thiobartituric acid-reactive substances; CAS, casein; SPI+, soya protein isolate; SPI-, soya protein isolate (negligible levels of phytochemicals); ACO, acyl-CoA oxidase; CPT-1, carnitine palmitoyltransferase I; HADHA, hydroxyacyl-CoA dehydrogenase/3-ketoacyl-CoA thiolase/enoyl-CoA hydratase; PPAR, peroxisome proliferator-activated receptors; CYP/A-1, cholesterol 7 alpha - hydroxylase; ABCG5, 8, ATP-binding cassette sub-family G members 5, 8; LXRa, liver X receptor alpha; EW, protein of egg white; SP, soya protein; fa/fa, obese phenotype; BW, body weight; 6-Keto PGF1a, 6-keto prostaglandin F1a; LIS, low isoflavone soya protein; HIS, high isoflavone soya protein; CR, casein + rosiglitazone; Co, cocoa; S, soya; O, oats; $\Omega$, fish oil, ASM, after metabolic syndrome; BSM, before metabolic syndrome; STD, standard diet; HFS, high-fat high-sucrose diet; Fen, fenugreek group; CON, starch diet; FRU, high-fructose diet; FRU + FPEt, high-fructose diet with fenugreek seed polyphenolic extract (200 mg/kg); FRU + Quer, high-

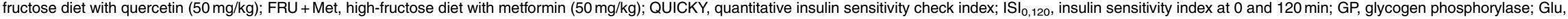
glucose; $I C D H$, isochrate dehylrogenase, SDH, succinale dehydrogenase, PTP, protein tyrosine phosphalases, PTK, protein tyrosine knases; FSP, tenugreek seed powder; Allx, alloxan, CHOL, cholestero, ALAT, alanine transaminase, ASAT, aspartate transaminase; ALP, alkaline phosphatase; GAL, galactomannan; NC, normal CT; Or, orlistat; MCC-PS, microcrystalline cellulose-potato starch; H, hyperlipidaemic; HMCC-PS, hyperlipidaemic diet and composite of MCC-PS; C, control; A, control $+1 \%$ adzuki bean; CF, high-fat diet; AF, high-fat diet $+1 \%$ adzuki bean; EtEx, ethanol extract of adzuki beans; PF, yellow pea fibre; PFL, yellow pea flour; PS, yellow pea starch; OFS, oligofructose; SREBP-1C, sterol regulatory element binding protein 1; ACC, acetyl-CoA carboxylase; WPF, whole pea flour; FPF, fractionated pea flour, LFO, licoride flavonoid oil, ND, normal diet, HFD, high-fat diet, LDP, low-dose pigeon pea; MDP, medium dose; HDP, high dose; PC, post-control; LDLr, LDL receptor; HMG-CoA, HMG-CoA reductase (3-hydroxy-3-methyl-glutaryl-CoA reductase); MDA, malondialdehyde; RCD, regular chow diet; HFD, high-fat diet; CSEE, Casia seed ethanol extract; PG, pioglitazone; AMPK, adenosine monophosphate protein kinase; WAT, white adipose tissue; CP, chickpea; L, lentils; AEPS, aqueous extract of $P$. santaniloides; MEPS, metanolic extract of $P$. santaniloides; Ovex, ovariectomised; F, fructose-fed rats; F-T, fructose-fed Tamarindus indica seed aqueous extract, TpALet, aqueous extract of Tephrosia purpurea leaves.

* References number ${ }^{200,25,30,35,31,39,37,45,46,48,56,60)}$ : no exact numeric data available, data represented by charts or figures.

$\dagger \mu \mathrm{mol}$ of glucose phosphorylated/h per $\mathrm{mg}$ protein.

$\mp \mu \mathrm{mol}$ of pyruvate formed/min per $\mathrm{mg}$ protein.

$\S \mu \mathrm{g}$ of Pi liberated/min per protein.

II $\mu \mathrm{mol}$ of Pi liberates $\mathrm{h}$ per protein.

If Total cholesterol:HDL-cholesterol ratio.
$\star \star$
LDL-cholesterol:HDL-cholesterol ratio. 
The interobserver raw agreement was calculated at $95 \cdot 12 \%$ $(k=0.725)$.

Mainly, as observed from the present review, the majority of the experiments were carried out using rats as an experimental model ( $n$ 30), followed by those that used mice ( $n$ 6). Focusing on the studies that used rats as the experimental model, different strategies for the induction and study of the MetS can be observed. Among them, the most common one is the induction of this pathology by diet in Wistar rats $(n 15)$ followed by its induction on Sprague-Dawley $(n 7)$ rats, another animal model that has been proven to be adequate for the study of this pathology. The most commonly used legume was Glycine max or soyabean ( $n$ 11), followed by Trigonella foenum gracecum or fenugreek ( $n$ 8) and Phaseolus vulgaris or beans $(n 4)$, whereas in the rest of the studies a variety of legumes was used. The most common form of legume administration was in the form of an extract ( $n$ 11) or protein/fibre flour $(n 7)$. It is worth mentioning that besides the study of the principal factors involved in the development of the MetS, the research is focused on the effects of the legume administration on the expression of several genes related to lipid, glucose and energy metabolism, as well as peptides and hormones associated with food intake, inflammatory markers and antioxidant status.

\section{Glycine max/soyabean}

Among the studies that used Glycine max as part of the diet intervention, one of them ${ }^{(17)}$ studied the effects of soyabean protein administration on pups of pregnant rats. The results of this study point out lower body weight and lipoprotein expression of the hepatic lipoprotein cytochrome P450, subfamily 2 , polypeptide 11 in the pups that consumed soya protein isolate. In addition, the specific intervention positively influenced genes involved in peroxisomal and mitochondrial fatty acid $\beta$-oxidation such as acyl-CoA oxidase ( $C O A)$, the mitochondrial trifunctional protein $\alpha$ subunit (hydroxyacyl-CoA dehydrogenase/3-ketoacyl-CoA thiolase/enoyl-CoA hydratase) and fatty acid transport into the mitochondria by carnitine palmitoyltransferase $1 \mathrm{~A}(C P T-1 A)$ by increasing their expression in the liver. Further improvements on hepatic and serum lipid metabolism parameters due to soyabean administration were described in other studies ${ }^{(18-23)}$. Specifically, among the mentioned studies, Barrios-Ramos et al. ${ }^{(18)}$ and Potu et al. ${ }^{(21)}$ indicated that the administration of powder and oil of soyabean induced improvements on hepatic steatosis and the hepatic inflammation marker c-reactive protein, respectively. In addition, proteins involved in lipid synthesis pathways (fatty acid synthase (FAS), acetyl-coenzyme A carboxylase $\alpha$, Stearoyl-CoA desaturase-1, fatty acid elongase 6, sterol regulatory element binding protein 1 (SREBP1) and carbohydrate-responsive element-binding protein) were down-regulated as a consequence of soyabean administration, thus suggesting an improvement in lipid metabolism pathways $^{(19,24)}$.

Regarding glucose metabolism, the majority of the studies suggest a clear improvement induced by the specific legume. A decrease in plasma glucose, leptin and insulin concentration, as well as an improvement in insulin sensitivity index $^{(17,18,20,24,25)}$, has been reported. Such a beneficial action of soyabean is further supported by increased expression of key enzymes and genes linked to glucose metabolism such as insulin I (INS1), insulin II (INS2), GLUT2 ${ }^{(20)}$ and PPAR $\alpha$ and $\operatorname{PPAR} \gamma^{(17,23,24)}$ in pancreas, liver, muscle and adipose tissue.

Two of the retrieved studies pointed out positive effects of Glycine max on blood pressure ${ }^{(18,26)}$, whereas Hwang et al. ${ }^{(27)}$ observed a decrease of renal glomerular size and the improvement in parameters associated with glomerular filtration in the groups of rats fed soya protein. In this regard, Davis et $a l^{(24)}$ and Palanisamy et $a l^{(26)}$ reported a lower kidney weight, urinary volume and creatinine concentration, as well as proteinuria, because of the administration of this legume in Zucker diabetic and Wistar rats with MetS, respectively. Regarding oxidative stress in this tissue, the levels of thiobarbituric acid-reactive substances (TBARS) and GSH were restored and brought back to normal levels after the administration of Glycine $\max ^{(26)}$.

The study of Zhou et al. ${ }^{(25)}$ focused on the effects of this legume on white adipose tissue, demonstrating a decrease of the weight of this tissue in male and female mice.

\section{Trigonella foenum gracecum/fenugreek}

The use of fenugreek in all its different forms - that is, seed powder $^{(28,29)}$, extract ${ }^{(30-32)}$ isolated polyphenols ${ }^{(33)}$ or polysaccharide galactomannan ${ }^{(34,35)}$ - points out to the beneficial changes in glucose metabolism, as demonstrated by lower levels of blood insulin, glucose, AUC, as well as higher homoeostatic model assessment for insulin resistance (HOMA-IR) index. Moreover, the re-establishment of the enzymes that play an integral role within the insulin signalling cascade back to normal levels highlights this potential action ${ }^{(33)}$. Specifically, Srichamroen et al. ${ }^{(35)}$ demonstrated that galactomannan of fenugreek reveals its function at the intestinal level by reducing the in vitro uptake of glucose in both jejunum and ileal segments. Moreover, the hypolipidaemic properties of fenugreek are clearly demonstrated by lower levels of lipid fractions in blood $^{(28-31,34)}$ and TAG in epidydimal adipose tissue $^{(34)}$, the weight of the latter being significantly lower after combining high-fat diets with powder of fenugreek seeds ${ }^{(28)}$. Liver function markers such as alanine transaminase (ALT), aspartate transaminase (AST) and alkaline phosphatase activities $^{(29,31)}$, concentration of TBARS, as well as the activities of antioxidant enzymes such as catalase and superoxide dismutase $^{(25)}$, decreased because of the administration of this legume. In addition, serum parameters of renal functionality such as urea, uric acid and creatinine were reduced by fenugreek extract administration ${ }^{(31)}$. Regarding the action of the specific legume on the immune system, Ramadan et al. ${ }^{(29)}$ investigated the effects of fenugreek seed powder using an immunosuppressive rat model and demonstrated its potential by decreasing abnormalities of the immune system such as leucopenia, neutropenia and lymphopenia while increasing spleen-weight:body weight ratio and cellularity of lymphoid organs. 


\section{Phaseolus vulgaris/beans}

The administration of $P$. vulgaris revealed a decrease in daily food intake and body weight, as well as improvements in plasma lipid parameters such as total cholesterol (TC), TAG, phospholipids and phosphorus phospholipids ${ }^{(36-38)}$. Moreover, bean consumption caused a decrease in acetyl-CoA carboxylase (ACC) and increments in cholesterol $7 \alpha$-hydroxylase levels ${ }^{\text {(38) }}$. Specifically, the study of Zaru et al. ${ }^{(39)}$ demonstrated a decrease in the seeking behaviour of chocolate-flavoured beverage of animals fed $P$. vulgaris extracts compared with the animals in the control group. Regarding plasma glucose metabolism parameters, only blood glucose, plasma leptin and AUC were determined, which were all lower after the administration of this legume ${ }^{(36,37)}$.

\section{Vigna angularis/adzuki beans}

In the three studies retrieved ${ }^{(40-42)}$, the dietary intervention with Vigna angularis/Adzuki beans included the administration of this legume as an extract. The studies focused on glucose and lipid metabolism, indicating a reduction in glucose, insulin, glycated $\mathrm{Hb}$ and microalbumin:creatinine ratio in the plasma of the animals. In addition, concentrations of TC, TAG, as well as lipid content of the liver, were reduced as a consequence of the administration of this legume. Similar reductions were produced in liver weight. In contrast, faecal weight and lipid excretion were found to be increased.

\section{Pissum sativum/yellow pea}

The two studies retrieved ${ }^{(43,44)}$ demonstrated a reduction in blood glucose and insulin concentrations due to yellow pea administration, as well as decreased hepatic TAG, decreased ACC and increased SREBP mRNA levels.

\section{Astragalus membranaceus/huáng-Qí} (translated as yellow leader)

The two studies retrieved ${ }^{(45,46)}$ used male diabetic animal models and aimed to study the effects of this legume on parameters related to glucose and lipid metabolism. Body weight was reduced resulting from legume administration, as well as parameters such as serum glucose and insulin concentrations, AUC and HOMA-IR index. In contrast, glucose infusion rate, after the performance of a hyperglycaemic clamp test, and hepatic glycogen content increased. Similar improvements were also found in parameters of lipid and energy metabolism represented by reduction of plasma TC and fatty acid concentration, as well as ACC and adenosine monophosphate activated protein (pAMPK) expression in the liver. The study of Gao et al. ${ }^{(45)}$ performed histology and immunohistochemistry analyses of pancreas, demonstrating reduced pathological changes, stain intensity and area in the groups administered with the legume. Inflammation markers studied by Hoo et $a l{ }^{(46)}$ were reduced in the adipose tissue of the treated groups.

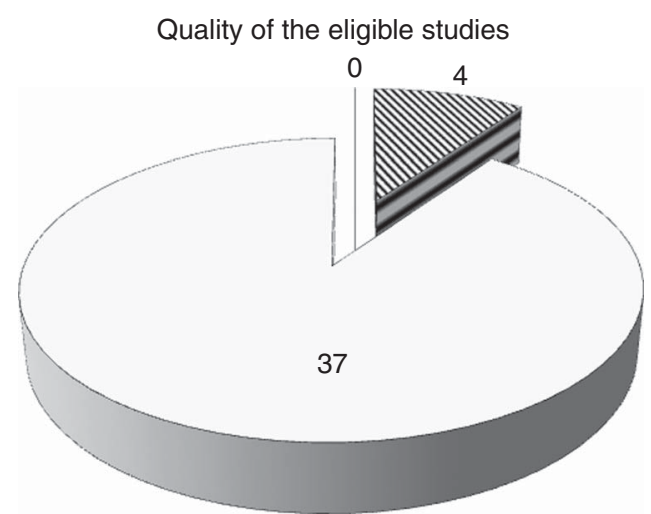

Fig. 2. Quality of the included studies of the systematic review. $\mathbb{\otimes}$, Excellent; $\square$, good; $\square$, bad.

\section{Glycyrrhiza glabra/liquorice}

The administration of Glycyrrbiza glabra lowered blood glucose, HOMA-IR index, serum insulin and leptin levels ${ }^{(47,48)}$. Moreover, the 18-week administration of liquorice flavonoid oil (LFO) (1\%) led to lower body weight and periuterine and white adipose tissue of female C7BL/6J mice, whereas LFO (2\%) decreased adipocyte diameter and number of lipid droplets. In addition, it caused the up-regulation of genes related to $\beta$-oxidation and acyl-CoA degradation and down-regulation of glycolytic lipogenesis genes and those associated with acetyl-CoA synthesis ${ }^{(47)}$. Increases in PPAR $\gamma$ and lipoprotein lipase (LPL) relative expressions after the administration of G. glabra were reported by the study of Yoke et $a l .{ }^{(48)}$.

\section{Other legumes}

Other legumes in addition to the previously described ones have shown different effects on parameters associated with the MetS. The administration of amorfrutins of Glycyrrbiza foetida and Amorpha fruticosa (false indigo ${ }^{(49)}$ ), Cajanus cajan (pigeon pea) powder, Pterocarpus santaniloides (Mututi ${ }^{(49)}$ ) leaf extract, Pueraria lobata (Kudzu ${ }^{(50)}$ ) root extract and Tamarindus indica (tamarind tree ${ }^{(51)}$ ) aqueous extract ${ }^{(52-56)}$ decreased blood glucose, insulin content, as well as glucose and insulin AUC. The above-mentioned legumes in addition to Tephrosia purpurea, Amorpha administrated as a leaf extract ${ }^{(57)}$ have also shown their beneficial effect on parameters of lipid metabolism by lowering the serum levels of different lipid fractions. Tzeng et al. ${ }^{(58)}$ demonstrated that an ethanol extract of Cassia tora (Foetid cassia ${ }^{(59)}$ ) reduced the size of white adipose tissue, as well as the expression of enzymes such as FAS and SREBP in this tissue. In addition, it up-regulated the expression pAMPK, pACC and CPT1, all enzymes related to energy metabolism, and improved parameters of cardiovascular function such as atherogenic index and coronary risk index. Focusing on hepatic lipid metabolism, legumes such as Aspalathus linearis (Roibos), Lens culinaris (Lentils), C. cajan, G. foetida and T. purpurea ${ }^{(52,56,60)}$ improved liver functionality by reducing liver weight, hepatic cholesterol and TAG content in addition to the reduction of lipid droplet accumulation and 
expression of TNF $\alpha$, a widely used inflammation marker. According to the results of the present systematic review, only one study by Peng et al. ${ }^{(54)}$ pointed out the beneficial effects on blood pressure after the inclusion of the root extract of $P$. lobata in the diet of the pups of an animal model of spontaneously hypertensive rats.

\section{Discussion}

The present systematic review was undertaken to give a comprehensive overview of the benefits of legume consumption on parameters related to the MetS and collect the existent mechanisms of action so far reported in animal experimental trials. In addition, it aimed to identify scarcities or abundancies with respect to legume consumption and its potential beneficial influence on the MetS alterations.

After the screening of the papers, data of forty-one studies were extracted. To our knowledge, this is the first systematic review gathering together the beneficial effects that a wide variety of legumes, most of them of common use, exert on the MetS, and include data on the way that legumes affect specific metabolic pathways involved in this pathology. The mechanistic emphasis of this review implies that preferentially animal studies were chosen.

Although some studies in humans indicate possible undesired effects due to the consumption of legumes, no such effects were reported in the studies collected for this review. Moreover, no toxicity effects by the administration of legumes in any form were reported. However, an increase of hepatic phospholipids was induced by the administration of adzuki beans $^{(41)}$, chickpeas and lentils ${ }^{(61)}$, in addition to a decrease of LPL activity in epididymal fat reported by the latter study. In addition, in the study of Shahraki et al. ${ }^{(55)}$, an elevation of AST and ALT was observed in the group that consumed the aqueous extract of $T$. indica. As for the insulin resistance, Wagner et $a l .{ }^{(23)}$ concluded that after soya isoflavone administration, insulin responses significantly increased and were accompanied by decreased plasma adiponectin concentrations. In a similar manner, administration of soyabean oil in Ossabaw pigs $^{(21)}$ resulted in elevated concentrations of glucose and insulin concentrations in plasma, as well as elevated blood lipids. Nevertheless, despite the negative effects of legume consumption in the above-mentioned studies, the majority of the studies gathered by the present systematic review highlight the beneficial effects of legume administration on the development and progression of the MetS and its related pathologies.

According to the results of the CACEC-EC questionnaire, the quality of the retrieved studies was good (Fig. 2), although there was great heterogeneity among them. In addition to the variety of legumes used, they were administered in different forms such as seed powder, extract or different fractions of the legume (protein, fibre). There was also great heterogeneity regarding the experimental period of the studies finally selected, which varied from $2 \mathrm{~h}^{(32)}$ to 40 weeks ${ }^{(23)}$. However, all of them were randomised intervention studies according to the inclusion criteria established.
The frequent use of Glycine max/soyabean in the studies retrieved can be explained because of the declaration of its protein as a good substitute for animal products, offering a 'complete' protein profile and its protective action against CVD $^{(62,63)}$ by the US Food and Drug Administration ${ }^{(64)}$. Most of the studies included the investigation of various metabolic parameters simultaneously trying to offer evidence on more than one metabolic pathway. The most widely mentioned parameters related to glucose, lipid and renal metabolism are included, whereas inflammation, oxidative status, blood pressure, body weight and body composition were studied in fewer studies. Only one study focused on the anorectic effects of legumes by reducing appetite and craving for food ${ }^{(39)}$

As impairments of glucose metabolism are directly related to the MetS, these alterations are widely studied. Therefore, lowering glucose concentration, HOMA-IR index or increasing insulin response are among the most reported findings. Such positive effects seem to be independent from the intervention duration, as even the shortest intervention ${ }^{(32)}$ induced an improvement in blood glucose. However, it is worth mentioning that in this study $T$. foenum graecum extract was directly injected in alloxan-induced diabetic animals. In general, twenty-nine of the retrieved studies showed improvements in glucose metabolism and included several legumes such as Glycine $\max ^{(17-20,24,26,38)}$, T. foenum $\operatorname{graecum}^{(28,29,31-33)}$, $P$. vulgaris ${ }^{(36-38)}, \quad V$. angularis ${ }^{(41)}$, Pisum sativum $^{(43,44)}$,', Astragalus membranaceus $^{(45,46)}$, G. glabra $^{(47,48)}$, C. cajan $^{(52)}$, G. foetida and A. fruticosa ${ }^{(56)}$, P. santaniloides ${ }^{(53)}$, P. lobata $^{(54)}$,' T. indica $^{(55)}$ and T. purpurea ${ }^{(57)}$. No such effects were reported for A. linearis $^{(60)}, C$. tora $^{(58)}$ and L. culinaris/Cicer arietinum $^{(61)}$. It seems that legumes influence the mechanistic pathways involving the expression of genes related to glucose metabolism such as GLUT2, GLUT4, INS1 or INS2 ${ }^{(20,24)}$, although the expression of more genes need to be studied. One of the retrieved studies also measured the activities of glucoseand glycogen-metabolising enzymes, therefore demonstrating the beneficial effect that polyphenols of $T$. foenum graecum exert on glucose metabolic pathways ${ }^{(33)}$. Moreover, the study of Srichamroen et al. ${ }^{(35)}$ revealed that another possible mechanism explaining glucose regulation is possible through the action of a galactomannan of the same legume in the reduction of the uptake of glucose by jejunum and ileal segments of the intestine.

In a manner similar to glucose metabolism, lipid parameters seem to be positively influenced by the administration of all sixteen different legumes that have been included in this review. Among the most widely mentioned beneficial improvements, the reduction of different lipid fractions in plasma, such as total-, LDL-, HDL-cholesterol and TAG $^{(20,22,28-31,34,36,38,46,48,54-56,58,61)}$, hepatic TAG and phospholipid content ${ }^{(17,19,60)}$, or both of them ${ }^{(18,24,40-43,45,52,61)}$, is reported. Other improvements associated with lipid metabolism and body composition are the decrease of body fat mass and white adipose tissue by Glycine $\max ^{(17,25)}$, as well as the reduction of hepatic steatosis induced by this same legume ${ }^{(18)}$. In this regard, the administration of G. glabra and A. linearis also reduced the number of lipid droplets in the liver ${ }^{(47,60)}$. Moreover, the studies of Aoki et al. ${ }^{(47)}$ and Tzeng et al. ${ }^{(58)}$ used 
the determination of mesenteric, perirenal, periuterine, inguinal and epidydimal fat as a marker of increased lipid adiposity in animals and further improvement of this parameter by the administration of G. glabra and C. tora, respectively. It is quite clear that the MetS is related to impaired fat excretion, whereas the administration of $V$. angularis extract ${ }^{(42)}$ and Pigeon pea ${ }^{(52)}$ improves such alteration. The results of the collected studies demonstrate that a great number of genes related to $\beta$-oxidation and acyl-CoA degradation are up-regulated by the administration of several legumes, whereas glycolytic lipogenesis genes are down-regulated. In particular, Glycine max ${ }^{(17,19,23,24)}$, P. vulgaris ${ }^{(38)}, \quad P$. sativum $^{(43)}$, A. membranaceus $^{(45)}$, G. glabra $^{(47,48)}, \quad$ C. cajan $^{(52)}$, C. tora ${ }^{(58)}$, G. foetida' A. fructicosa ${ }^{(56)}$ are among the encountered legumes with such action. Still, collected data indicate that more research needs to be developed on these and other potential mechanism related to the beneficial influence of legumes on lipid metabolism, whereas a greater range of legume species needs to be tested.

It is well known that renal alterations can occur with the development of the MetS. However, as demonstrated by the results of this systematic review, only six of the collected studies mention beneficial results on renal metabolism in which only four different legumes are included: Glycine $\max ^{(24,26,27)}$, T. foenum graecum $^{(31)}$, V. angularis ${ }^{(41)}$ and C. cajan $^{(52)}$. In this regard, legume administration managed to restore the augmented kidney weight, urea level, uric acid and creatinine derived from the administration of a high-fructose diet. The presence of glucose and protein in urine are also linked to alterations of renal metabolism and were improved by the administration of $V$. angularis ${ }^{(41)}$ and Glycine max ${ }^{(24)}$. Worth mentioning is the study by Palanisamy et $a l{ }^{(26)}$ that described a simultaneous reduction of blood pressure together with concomitant improvements in renal metabolism, as soya protein reduced glucose levels and produced the inhibition of the angiotensin-converting enzyme. Still, there is a lack of information in this field for the majority of the legumes gathered by this review.

The process of inflammation is highly involved in the development of the MetS and can be determined by the concentration of oxidative markers or the activity of antioxidant enzymes in different organs. As observed by this systematic review, only five of the legumes collected have been so far used to investigate these parameters. Among them, Glycine $\max ^{(21,26)}$, T. foenum graceum $^{(29,30)}$, A. membranaceus ${ }^{(46)}$, C. cajan $^{(52)}$ and Glythirrbiza foetida/A. fructicosa ${ }^{(56)}$ are encountered. Two clear tendencies are observed for the evaluation of these parameters: on the one hand, the simultaneous determination of oxidative damage, as well as antioxidant enzymes ${ }^{(26,30,52)}$, and on the other hand ${ }^{(29,46,56)}$, the study of the level of cytokines involved in the process of inflammation.

Overall, legume administration positively affects glucose and lipid metabolism, which include the most widely studied parameters. Fewer studies have been focused in renal metabolism and the properties of legumes as antioxidant and antiinflammatory agents. A possible limitation of the present review is that the bibliographic search was carried out based on the definition of search terms through the use of MeSH, not followed by all studies. It is important that the same rules be followed for the establishment of key words so that the inclusion of all available studies would be ensured.

\section{Acknowledgements}

This study was funded by grant P09-AGR-4658 from Junta de Andalucía, Spain. The authors also want to acknowledge the Ministry of Economy and Competitiveness (MINECO, Spain) for the concession of the projects AGL2013-43247-R and DEP201458296-R and the European Union for financing the FEDER programme.

G. K., R. M., J. M. P. and M. L.-J. designed the research; R. M. and G. K. conducted the research; J. M. P. and M. L.-J. revised the papers when consensus was needed; C. W.-B. and J. S.-V. provided useful guidelines for the methodology followed for the systematic review; R. M. and G. K. wrote the paper. All authors of this manuscript share responsibility for its final content. All authors read and approved the final manuscript.

The authors declare no conflicts of interest arising from the conclusions of this work.

\section{References}

1. Anderson PJ, Critchley JAJH, Chan JCN, et al. (2001) Factor analysis of the metabolic syndrome: obesity vs insulin resistance as the central abnormality. Int J Obes Relat Metab Disord 25, 1782-1788.

2. Indulekha K, Surendar J \& Mohan V (2011) High sensitivity C-reactive protein, tumor necrosis factor- $\alpha$, interleukin- 6 , and vascular cell adhesion molecule-1 levels in Asian Indians with metabolic syndrome and insulin resistance (CURES-105). J Diabetes Sci Technol 5, 982-988.

3. International Diabetes Federation (2006) IDF worldwide definition of the metabolic syndrome. http://www.idf.org/ metabolic-syndrome (accessed October 2014).

4. Reaven GM (1988) Role of insulin resistance in human disease. Diabetes 37, 1595-1607.

5. Alberti K, Zimmet P \& Shaw J (2006) Metabolic syndrome a new world-wide definition. A Consensus Statement from the International Diabetes Federation. Diabet Med 23, 469-480.

6. Kaur J (2014) A comprehensive review on metabolic syndrome. Cardiol Res Pract 2014, e943162.

7. Manco M, Bottazzo G, DeVito R, et al. (2008) Nonalcoholic fatty liver disease in children. J Am Coll Nutr 27, 667-676.

8. Deen D (2004) Metabolic syndrome: time for action. Am Fam Physician 69, 2875-2882.

9. Duranti M (2006) Grain legume proteins and nutraceutical properties. Fitoterapia $\mathbf{7 7}, 67-82$

10. König D, Muser K, Berg A, et al. (2012) Fuel selection and appetite-regulating hormones after intake of a soy proteinbased meal replacement. Nutrition 28, 35-39.

11. Segura Campos MR, Chel Guerrero LA \& Betancur Ancona DA (2010) Angiotensin-I converting enzyme inhibitory and antioxidant activities of peptide fractions extracted by ultrafiltration of cowpea Vigna unguiculata hydrolysates. J Sci Food Agric 90, 2512-2518.

12. Yoshie-Stark Y \& Wäsche A (2004) In vitro binding of bile acids by lupin protein isolates and their hydrolysates. Food Chem 88, 179-184. 
13. Tomás-Casterá V, Sanz-Valero J \& Juan-Quilis V (2013) [Bibliometric analysis of the scientific production and consumption on nutrition journals indexed in scielo network]. Nutr Hosp 28, 969-970.

14. Burton RE \& Kebler RW (1960) The 'half-life' of some scientific and technical literatures. Am Doc 11, 18-22.

15. Cohen J (1968) Weighted kappa: nominal scale agreement provision for scaled disagreement or partial credit. Psychol Bull 70, 213-220.

16. Wanden-Berghe C \& Sanz-Valero J (2012) Systematic reviews in nutrition: standardized methodology. Br J Nutr 107, S3-S7.

17. Ronis MJ, Chen Y, Badeaux J, et al. (2009) Dietary soy protein isolate attenuates metabolic syndrome in rats via effects on PPAR, LXR, and SREBP signaling. J Nutr 139, 1431-1438.

18. Barrios-Ramos JP, Garduño-Siciliano L, Loredo M, et al. (2012) The effect of cocoa, soy, oats and fish oil on metabolic syndrome in rats. J Sci Food Agric 92, 2349-2357.

19. Mori T, Kondo H, Hase T, et al. (2011) Dietary phospholipids ameliorate fructose-induced hepatic lipid and metabolic abnormalities in rats. J Nutr 141, 2003-2009.

20. Nordentoft I, Jeppesen PB, Hong J, et al. (2008) Increased insulin sensitivity and changes in the expression profile of key insulin regulatory genes and beta cell transcription factors in diabetic KKAy-mice after feeding with a soy bean protein rich diet high in isoflavone content. J Agric Food Chem 56, 4377-4385.

21. Potu RB, Lu H, Adeola O, et al. (2013) Metabolic markers in Ossabaw pigs fed high fat diets enriched in regular or low $\alpha$-linolenic acid soy oil. Nutr Metab 10, 27.

22. Torre-Villalvazo I, Tovar AR, Ramos-Barragán VE, et al. (2008) Soy protein ameliorates metabolic abnormalities in liver and adipose tissue of rats fed a high fat diet. J Nutr 138, 462-468.

23. Wagner JD, Zhang L, Shadoan MK, et al. (2008) Effects of soy protein and isoflavones on insulin resistance and adiponectin in male monkeys. Metabolism 57, Suppl. 1, S24-S31.

24. Davis J, Higginbotham A, O'Connor T, et al. (2007) Soy protein and isoflavones influence adiposity and development of metabolic syndrome in the obese male ZDF rat. Ann Nutr Metab 51, 42-52.

25. Zhou J-R, Li L \& Pan W (2007) Dietary soy and tea combinations for prevention of breast and prostate cancers by targeting metabolic syndrome elements in mice. Am J Clin Nutr 86, s882-s888.

26. Palanisamy N, Viswanathan P, Ravichandran MK, et al. (2010) Renoprotective and blood pressure-lowering effect of dietary soy protein via protein kinase $C \beta I I$ inhibition in a rat model of metabolic syndrome. Can J Physiol Pharmacol 88, 28-37.

27. Hwang S-Y, Taylor CG, Zahradka P, et al. (2008) Dietary soy protein reduces early renal disease progression and alters prostanoid production in obese $\mathrm{fa} / \mathrm{fa}$ Zucker rats. $J$ Nutr Biochem 19, 255-262.

28. Muraki E, Chiba H, Tsunoda N, et al. (2011) Fenugreek improves diet-induced metabolic disorders in rats. Horm Metab Res 43, 950-955.

29. Ramadan G, El-Beih NM \& Abd El-Kareem HF (2011) Anti-metabolic syndrome and immunostimulant activities of Egyptian fenugreek seeds in diabetic/obese and immunosuppressive rat models. Br J Nutr 105, 995-1004.

30. Belguith-Hadriche O, Bouaziz M, Jamoussi K, et al. (2010) Lipid-lowering and antioxidant effects of an ethyl acetate extract of fenugreek seeds in high-cholesterol-fed rats. J Agric Food Chem 58, 2116-2122.

31. Eidi A, Eidi M \& Sokhteh M (2007) Effect of fenugreek (Trigonella foenum-graecum $\mathrm{L}$ ) seeds on serum parameters in normal and streptozotocin-induced diabetic rats. Nutr Res 27, 728-733.
32. Mowla A, Alauddin M, Rahman MA, et al. (2009) Antihyperglycemic effect of Trigonella foenum-graecum (fenugreek) seed extract in alloxan-induced diabetic rats and its use in diabetes mellitus: a brief qualitative phytochemical and acute toxicity test on the extract. Afr J Tradit Complement Altern Med 6, 255-261.

33. Kannappan S \& Anuradha CV (2009) Insulin sensitizing actions of fenugreek seed polyphenols, quercetin \& metformin in a rat model. Indian J Med Res 129, 401-408.

34. Srichamroen A, Field CJ, Thomson ABR, et al. (2008) The modifying effects of galactomannan from Canadian-grown fenugreek (Trigonella foenum-graecum L.) on the glycemic and lipidemic status in rats. J Clin Biochem Nutr 43, 167-174.

35. Srichamroen A, Thomson ABR, Field CJ, et al. (2009) In vitro intestinal glucose uptake is inhibited by galactomannan from Canadian fenugreek seed (Trigonella foenum graecum $\mathrm{L}$ ) in genetically lean and obese rats. Nutr Res 29, 49-54.

36. Adel AM \& El-shinnawy NA (2012) Hypolipidemic applications of microcrystalline cellulose composite synthesized from different agricultural residues. Int J Biol Macromol 51, 1091-1102.

37. Carai MAM, Fantini N, Loi B, et al. (2011) Multiple cycles of repeated treatments with a Phaseolus vulgaris dry extract reduce food intake and body weight in obese rats. $\mathrm{Br} J \mathrm{Nutr}$ 106, $762-768$.

38. Zhu Z, Jiang W \& Thompson HJ (2012) Edible dry bean consumption (Phaseolus vulgaris L.) modulates cardiovascular risk factors and diet-induced obesity in rats and mice. Br J Nutr 108, Suppl. 1, S66-S73.

39. Zaru A, Maccioni P, Riva A, et al. (2013) Reducing effect of a combination of Phaseolus vulgaris and Cynara scolymus extracts on operant self-administration of a chocolateflavoured beverage in rats. Phytother Res PTR 27, 944-947.

40. Itoh T \& Furuichi Y (2009) Lowering serum cholesterol level by feeding a 40\% ethanol-eluted fraction from HP-20 resin treated with hot water extract of adzuki beans (Vigna angularis) to rats fed a high-fat cholesterol diet. Nutrition 25, 318-321.

41. Itoh T, Kobayashi M, Horio F, et al. (2009) Hypoglycemic effect of hot-water extract of adzuki (Vigna angularis) in spontaneously diabetic KK-A(y) mice. Nutrition 25, 134-141.

42. Kitano-Okada T, Ito A, Koide A, et al. (2012) Anti-obesity role of adzuki bean extract containing polyphenols: in vivo and in vitro effects. J Sci Food Agric 92, 2644-2651.

43. Eslinger AJ, Eller LK \& Reimer RA (2014) Yellow pea fiber improves glycemia and reduces Clostridium leptum in diet-induced obese rats. Nutr Res 34, 714-722.

44. Marinangeli CPF, Krause D, Harding SV, et al. (2011) Whole and fractionated yellow pea flours modulate insulin, glucose, oxygen consumption, and the caecal microbiome in Golden Syrian hamsters. Appl Physiol Nutr Metab 36, 811-820.

45. Gao L, Liu Q, Liu S, et al. (2014) A refined-JinQi-JiangTang tablet ameliorates prediabetes by reducing insulin resistance and improving beta cell function in mice. J Ethnopharmacol 151, 675-685.

46. Hoo RL, Wong JY, Qiao CF, et al. (2010) The effective fraction isolated from Radix Astragali alleviates glucose intolerance, insulin resistance and hypertriglyceridemia in $\mathrm{db} / \mathrm{db}$ diabetic mice through its anti-inflammatory activity. Nutr Metab 7, 67.

47. Aoki F, Honda S, Kishida H, et al. (2007) Suppression by licorice flavonoids of abdominal fat accumulation and body weight gain in high-fat diet-induced obese C57BL/6J mice. Biosci Biotechnol Biochem 71, 206-214.

48. Yoke Yin C, So Ha T \& Abdul Kadir K (2010) Effects of glycyrrhizic acid on peroxisome proliferator-activated receptor gamma (PPARgamma), lipoprotein lipase (LPL), serum lipid and HOMA-IR in rats. PPAR Res 2010, 530265. 
49. International Legume Database \& Information Service (2015) ILDIS home page. http://www.ildis.org/ (accessed March 2016).

50. Ulbricht C, Costa D, Dam C, et al. (2015) An evidence-based systematic review of Kudzu (Pueraria lobata) by the Natural Standard Research Collaboration. J Diet Suppl 12, 36-104.

51. Kuru P (2014) Tamarindus indica and its health related effects. Asian Pac J Trop Biomed 4, 676-681.

52. Dai F-J, Hsu W-H, Huang J-J, et al. (2013) Effect of pigeon pea (Cajanus cajan L.) on high-fat diet-induced hypercholesterolemia in hamsters. Food Chem Toxicol 53, 384-391.

53. Okwuosa CN, Unekwe PC, Achukwu PU, et al. (2013) Glucose and triglyceride lowering activity of Pterocarpus santaniloides leaf extracts against dexamethasone induced hyperlipidemia and insulin resistance in rats. Afr J Biotechnol 10, 9415-9420.

54. Peng N, Prasain JK, Dai Y, et al. (2009) Chronic dietary kudzu isoflavones improve components of metabolic syndrome in stroke-prone spontaneously hypertensive rats. I Agric Food Chem 57, 7268-7273.

55. Shahraki MR, Harati M \& Shahraki AR (2011) Prevention of high fructose-induced metabolic syndrome in male Wistar rats by aqueous extract of Tamarindus indica seed. Acta Med Iran 49, 277-283.

56. Weidner C, de Groot JC, Prasad A, et al. (2012) Amorfrutins are potent antidiabetic dietary natural products. Proc Natl Acad Sci 109, 7257-7262.

57. Pavana P, Manoharan S, Renju GL, et al. (2007) Antihyperglycemic and antihyperlipidemic effects of Tephrosia purpurea leaf extract in streptozotocin induced diabetic rats. J Environ Biol 28, 833-837.

58. Tzeng T-F, Lu H-J, Liou S-S, et al. (2013) Reduction of lipid accumulation in white adipose tissues by Cassia tora (Leguminosae) seed extract is associated with AMPK activation. Food Chem 136, 1086-1094.

59. Awasthi VK, Mahdi F, Chander R, et al. (2015) Hypolipidemic activity of Cassia tora seeds in hyperlipidemic rats. Indian J Clin Biochem 30, 78-83.

60. Beltrán-Debón R, Rull A, Rodríguez-Sanabria F, et al. (2011) Continuous administration of polyphenols from aqueous rooibos (Aspalathus linearis) extract ameliorates dietaryinduced metabolic disturbances in hyperlipidemic mice. Phytomedicine 18, 414-424.

61. Boualga A, Prost J, Taleb-Senouci D, et al. (2009) Purified chickpea or lentil proteins impair VLDL metabolism and lipoprotein lipase activity in epididymal fat, but not in muscle, compared to casein, in growing rats. Eur J Nutr $\mathbf{4 8}$, 162-169.

62. Sirtori CR, Gatti E, Mantero O, et al. (1979) Clinical experience with the soybean protein diet in the treatment of hypercholesterolemia. Am J Clin Nutr 32, 1645-1658.

63. Descovich GC, Gaddi A, Mannino G, et al. (1980) Multicentre study of soybean protein diet for outpatient hypercholesterolaemic patients. Lancet ii, 709-712.

64. Sacks FM, Lichtenstein A, Horn LV, et al. (2006) Soy protein, isoflavones, and cardiovascular health: an American Heart Association Science Advisory for Professionals from the Nutrition Committee. Circulation 113, 1034-1044. 